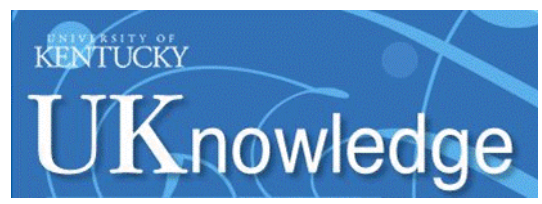

University of Kentucky

UKnowledge

$11-17-2021$

\title{
Adult Spiny Mice (Acomys) Exhibit Endogenous Cardiac Recovery in Response to Myocardial Infarction
}

\author{
Hsuan Peng \\ University of Kentucky, Hsuan.Peng@uky.edu \\ Kazuhiro Shindo \\ University of Kentucky, Kazuhiro.Shindo@uky.edu \\ Renée R. Donahue \\ University of Kentucky, renee.donahue@uky.edu \\ Erhe Gao \\ Temple University \\ Brooke M. Ahern \\ University of Kentucky, brooke.ahern@gmail.com
}

Sellowext this and fadditionahwworksat; https://uknowledge.uky.edu/physiology_facpub

Part of the Cardiovascular System Commons, Internal Medicine Commons, Physiology Commons, and the Translational Medical Research Commons

Right click to open a feedback form in a new tab to let us know how this document benefits you.

\section{Repository Citation}

Peng, Hsuan; Shindo, Kazuhiro; Donahue, Renée R.; Gao, Erhe; Ahern, Brooke M.; Levitan, Bryana M.; Tripathi, Himi; Powell, David; Noor, Ahmed; Elmore, Garrett A.; Satin, Jonathan; Seifert, Ashley W.; and Abdel-Latif, Ahmed K., "Adult Spiny Mice (Acomys) Exhibit Endogenous Cardiac Recovery in Response to Myocardial Infarction" (2021). Physiology Faculty Publications. 185.

https://uknowledge.uky.edu/physiology_facpub/185

This Article is brought to you for free and open access by the Physiology at UKnowledge. It has been accepted for inclusion in Physiology Faculty Publications by an authorized administrator of UKnowledge. For more information, please contact UKnowledge@lsv.uky.edu. 


\title{
Adult Spiny Mice (Acomys) Exhibit Endogenous Cardiac Recovery in Response to Myocardial Infarction
}

\author{
Digital Object Identifier (DOI) \\ https://doi.org/10.1038/s41536-021-00186-4
}

\section{Notes/Citation Information}

Published in npj Regenerative Medicine , v. 6, article no. 74.

\section{(C) 2021 The Author(s)}

This article is licensed under a Creative Commons Attribution 4.0 International License, which permits use, sharing, adaptation, distribution and reproduction in any medium or format, as long as you give appropriate credit to the original author(s) and the source, provide a link to the Creative Commons license, and indicate if changes were made. The images or other third party material in this article are included in the article's Creative Commons license, unless indicated otherwise in a credit line to the material. If material is not included in the article's Creative Commons license and your intended use is not permitted by statutory regulation or exceeds the permitted use, you will need to obtain permission directly from the copyright holder. To view a copy of this license, visit https://creativecommons.org/licenses/by/4.0/.

\section{Authors}

Hsuan Peng, Kazuhiro Shindo, Renée R. Donahue, Erhe Gao, Brooke M. Ahern, Bryana M. Levitan, Himi Tripathi, David Powell, Ahmed Noor, Garrett A. Elmore, Jonathan Satin, Ashley W. Seifert, and Ahmed K. Abdel-Latif 
ARTICLE OPEN recovery in response to myocardial infarction

Hsuan Peng ${ }^{1,8}$, Kazuhiro Shindo $\mathbb{D}^{1,8}$, Renée R. Donahue ${ }^{1}{ }^{1}$, Erhe Gao ${ }^{2}$, Brooke M. Ahern ${ }^{3}$, Bryana M. Levitan ${ }^{3,4}$, Himi Tripathi ${ }^{1}$, David Powell ${ }^{4}$, Ahmed Noor ${ }^{4}$, Garrett A. Elmore ${ }^{3}$, Jonathan Satin ${ }^{3 凶}$, Ashley W. Seifert $\mathbb{1}^{5 凶}$ and Ahmed Abdel-Latif ${ }^{1,4,6,7 凶}$

Complex tissue regeneration is extremely rare among adult mammals. An exception, however, is the superior tissue healing of multiple organs in spiny mice (Acomys). While Acomys species exhibit the remarkable ability to heal complex tissue with minimal scarring, little is known about their cardiac structure and response to cardiac injury. In this study, we first examined baseline Acomys cardiac anatomy and function in comparison with commonly used inbred and outbred laboratory Mus strains (C57BL6 and CFW). While our results demonstrated comparable cardiac anatomy and function between Acomys and Mus, Acomys exhibited a higher percentage of cardiomyocytes displaying distinct characteristics. In response to myocardial infarction, all animals experienced a comparable level of initial cardiac damage. However, Acomys demonstrated superior ischemic tolerance and cytoprotection in response to injury as evidenced by cardiac functional stabilization, higher survival rate, and smaller scar size 50 days after injury compared to the inbred and outbred mouse strains. This phenomenon correlated with enhanced endothelial cell proliferation, increased angiogenesis, and medium vessel maturation in the peri-infarct and infarct regions. Overall, these findings demonstrate augmented myocardial preservation in spiny mice post-MI and establish Acomys as a new adult mammalian model for cardiac research.

npj Regenerative Medicine (2021)6:74; https://doi.org/10.1038/s41536-021-00186-4

\section{INTRODUCTION}

Significant clinical advances for heart revascularization and nextgeneration medical therapies have improved the mortality rate after myocardial infarction (MI) ${ }^{1}$. The initial ischemic insult, even with timely revascularization, is followed by microvascular injury and infarct expansion leading to exacerbated damage ${ }^{2}$. Unfortunately, no therapies exist to limit myocardial damage from $\mathrm{MI}$ and the ensuing infarct expansion results in millions of recovering patients progressing to develop heart failure (HF). This is because the normal healing response to tissue injury in adult mammals is fibrotic repair; an effective short-term strategy, but one that leads to compromised tissue function in the long-term. Fibrotic repair is the default strategy in the adult heart where it is the leading cause of the clinical HF epidemic ${ }^{3}$. Multiple therapies developed for limiting infarct expansion or cardiac repair based on animal models have achieved only modest clinical success. A failure to translate basic research findings into successful clinical treatments can be traced, in part, to a lack of adult mammalian models possessing either ventricular regeneration or the ability for myocardial preservation.

In contrast to humans ${ }^{4-6}$, the ability to regenerate injured organs is widespread among vertebrates. Fishes, newts, and salamanders have extensive regenerative ability, and can functionally replace heart tissue after amputation or severe injury by expediting revascularization ${ }^{7}$ and by mobilizing a highly proliferative cardiomyocyte $(\mathrm{CM})$ pool $^{8,9}$. Specifically, zebrafish are a well-characterized model for adult cardiac regeneration with the documented ability to recover from a plethora of heart injuries including apical resection ${ }^{10}$, cryo-injury ${ }^{11}$, and coronary artery ligation. Neonatal mice are also capable of limiting the initial damage and repairing the myocardium after excision and ischemic injury during the first few days after birth ${ }^{12}$. In contrast, most adult mammals generally exhibit poor recovery capacity, especially as it pertains to recovering from heart damage. Spiny mice (Acomys spp.) are murid rodents found throughout Africa, the Middle East and Western Asia. These rodents exhibit a number of special traits; ${ }^{13}$ tantamount among them is the ability to regenerate skin, complex tissue ${ }^{14-19}$, and nephric tissue ${ }^{20}$. Recently, a large-scale survey of mammals suggested that spiny mice CMs exhibit a higher incidence of diploidy relative to laboratory mice ${ }^{21}$, while another study reported enhanced recovery after infarction injury ${ }^{22}$. Still, it remains poorly understood how spiny mice respond to heart injury and the mechanistic basis for their recovery remains unknown.

To rigorously examine how spiny mice respond to heart damage, we first detail Acomys heart structure and cardiac function in comparison to the most widely used inbred laboratory Mus model of cardiac injury (C57BL6). For comparative purposes we also used an outbred Swiss Webster strain from Charles River (CFW) to account for larger size and greater genetic diversity within our spiny mouse population. This cross-species characterization indicates similar cardiac structure and comparable baseline function among spiny mice and the examined mouse strains. We next conducted a comparative analysis of these animals in response to permanent coronary artery ligation (MI). In contrast to the mouse strains studied, Acomys showed significantly improved survival and enhanced myocardial preservation following ischemic injury. The ability of Acomys to recover from ischemic

\footnotetext{
${ }^{1}$ Saha Cardiovascular Research Center, College of Medicine, University of Kentucky, Lexington, KY, USA. ${ }^{2}$ The Center for Translational Medicine, Lewis Katz School of Medicine, Temple University, Philadelphia, PA, USA. ${ }^{3}$ Department of Physiology, College of Medicine, University of Kentucky, Lexington, KY, USA. ${ }^{4}$ Gill Heart and Vascular Institute and Division of Cardiovascular Medicine, University of Kentucky, Lexington, KY, USA. ${ }^{5}$ Department of Biology, University of Kentucky, Lexington, KY, USA. ${ }^{6}$ The Lexington VA Medical Center, Lexington, KY, USA. ${ }^{7}$ Division of Cardiovascular Medicine, Department of Internal Medicine, University of Michigan, Ann Arbor, MI, USA. ${ }^{8}$ These authors contributed equally: Hsuan Peng, Kazuhiro Shindo ${ }^{凶}$ email: Jonathan.Satin@uky.edu; awseifert@uky.edu; aalatif@umich.edu
} 
a

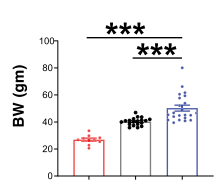

f

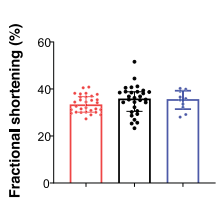

b
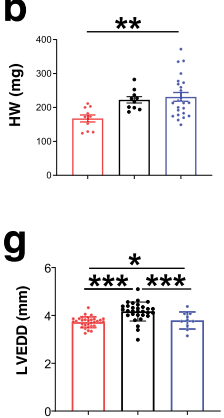

C

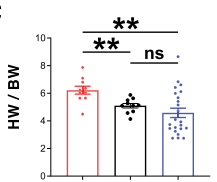

h

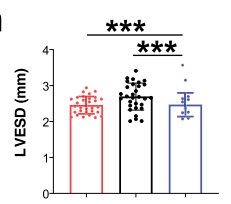

d

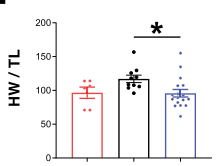

i

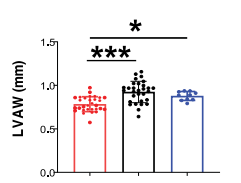

e

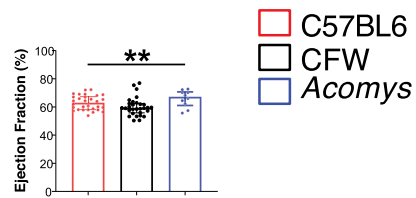

j

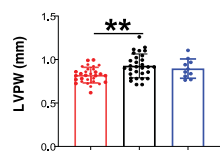

k

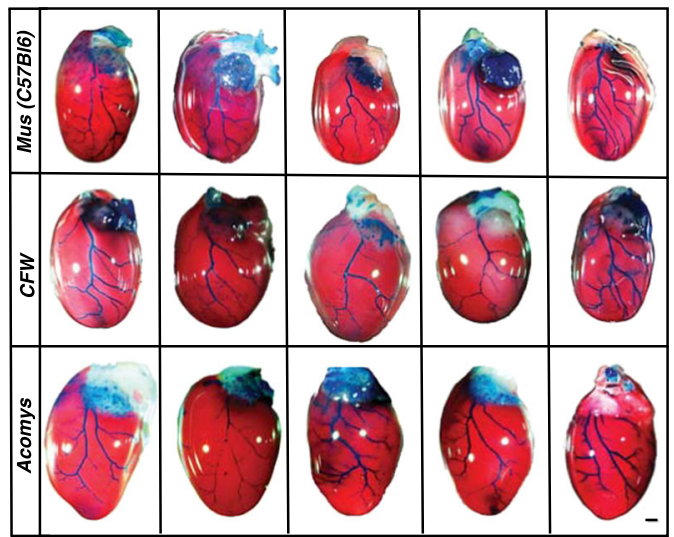

\begin{tabular}{|c|c|c|c|c|}
\hline $\begin{array}{c}3 \\
x+y\end{array}$ & 武地 & $\left.\begin{array}{l}3 \\
3 \\
3\end{array}\right\}$ & 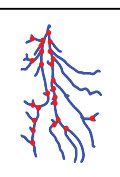 & (j) \\
\hline te & th & 称 & & (s) \\
\hline 数高 & $41)$ & $\pi^{\pi}$ & 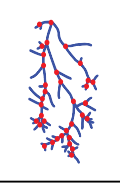 & ? \\
\hline
\end{tabular}

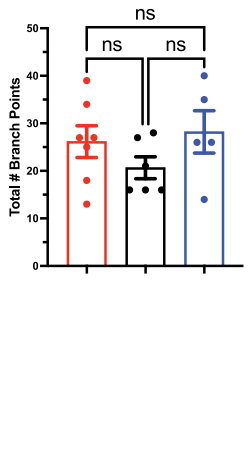

Fig. 1 Characteristics of Acomys heart physiology and anatomy at baseline. a-j Measures of body weight (BW) and heart weight in C57BL6Mus $(N=10), C F W-M u s(N=19)$, and Acomys $(N=22)$. Analyses demonstrate comparable heart weight across species when normalized by body weight $(\mathrm{BW})$ and tibia length (TL) (values are means \pm S.E.M, ${ }^{*} P<0.05,{ }^{* *} P<0.01$, and ${ }^{* * *} P<0.001$ by one-way ANOVA and Dunnett correction with Acomys as control). Ejection fraction $\left(F=4.1,{ }^{*} P<0.05\right)(\mathbf{e})$, fractional shortening $(F=0.96, P>0.05)(\mathbf{f})$, left ventricular enddiastolic diameter (LVEDD) $\left(F=14.8,{ }^{* *} P<0.01\right)(\mathbf{g})$, left ventricular end-systolic diameter (LVESD) $(F=4.6)(\mathbf{h})$, left ventricular anterior wall (LVAW) $\left(F=14.9,{ }^{*} P<0.05\right)$ (i), left ventricular posterior wall (LVPW) $(F=6.4)(j)(N=30$ C57BL6-Mus, 10 CFW-Mus and 10 Acomys). Representative images showing comparable left anterior descending artery coronary anatomy across species using intra-aortic infusion of Batson's 17 polymer mixture and corrosion casting $(N=5$ each group, scale bar $=1 \mathrm{~mm})(\mathbf{k})$. Quantification of coronary branching points of the arterial supply of the left ventricle across species. This analysis shows a comparable number of branching points across species with numerically higher (but not statistically significant) branching points in C57BL6-Mus and Acomys compared to CFW-Mus (I). Detailed statistical output for this figure can be found in the Supplementary Information.

injury is coincident with greatly improved angiogenesis rarely seen in adult mammals. These findings in Acomys support a rapid angiogenic response into fibrotic tissue which limits scar spread and enhances ischemic tolerance in response to MI.

\section{RESULTS}

\section{Heart and coronary tree anatomy are similar among Acomys and Mus (C57BL6 and CFW)}

To assess whether interpretable comparisons could be made across species, we first characterized hearts from Acomys and the most commonly used laboratory mouse (Mus) strain: C57BL6. In addition, we included an outbred Swiss Webster strain from Charles River (CFW) to control for increased size and genetic diversity in Acomys. To control for lifespan differences between species, we used sexually mature males, 6-month-old Acomys, and 8-12 week old Mus for these studies. First, we set out to determine whether baseline differences in cardiac gravimetrics exist between Acomys and Mus (C57BL6 and CFW). We assessed heart weight $(\mathrm{HW})$ and normalized it to body size (body weight [BW] and tibia length [TL]) to account for overall size differences between species (Fig. 1a-d, and Supplementary Table 1). Although Acomys demonstrated a higher mean HW compared to C57BL6 (Fig. 1b), normalized to BW, Acomys was not significantly different compared to CFW and exhibited only a slightly lower HW/BW compared to C57BL6 (Fig. 1c). CFW mice demonstrated a slightly higher HW compared to Acomys and C57BL6 when normalized to TL (Fig. 1d). Acomys showed heavier wet lung weight compared to Mus (C57BL6 and CFW) (Supplementary Fig. 1a), but lower wet and dry lung weight after normalization by BW (Supplementary Fig. 1b, e). Importantly, dry lung weight before normalization by TL and dry and wet lung weight after normalization by TL were not significantly different across species (Supplementary Fig. 1d-f). The complete data is summarized in Supplementary Table 1.

Next, we compared cardiac functional parameters and observed comparable baseline cardiac function under physiological conditions across species. Global cardiac function as presented by left ventricular ejection fraction was statistically higher in Acomys compared to Mus (Fig. 1e), but fractional shortening was not significantly different across species (Fig. 1f). Measures of left ventricular cavity diameter were also comparable between species, with the exception of CFW-Mus showing slightly larger left ventricular end-diastolic diameter and thicker anterior wall compared to Acomys (Fig. 1g-j and Supplementary Table 1). We also assessed gross cardiac structure and coronary tree anatomy among Acomys and Mus (C57BL6 and CFW) and found that cardiac structure was not grossly different between the species upon visual comparison of whole hearts along the short- or long-axis (Supplementary Fig. 1g). Using corrosion casting and intra-aortic infusion of Batson's 17 polymer mixture, we delineated the left 
a
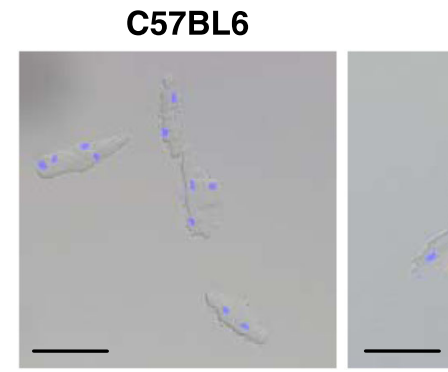

CFW
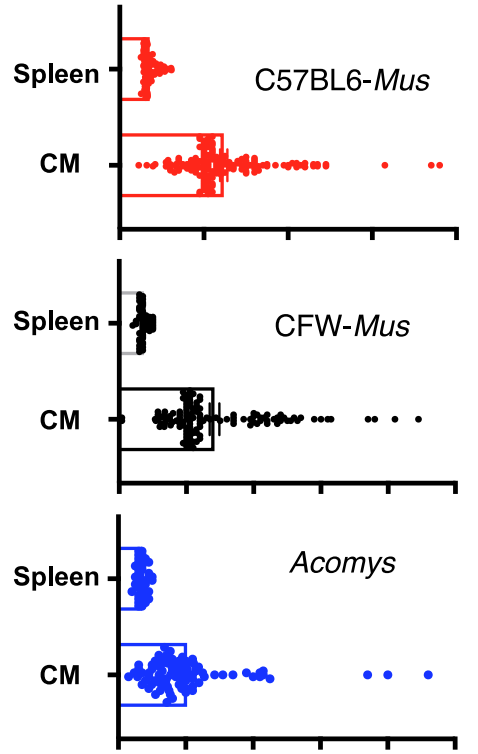

DAPI Fluorescence Intensity (AU)
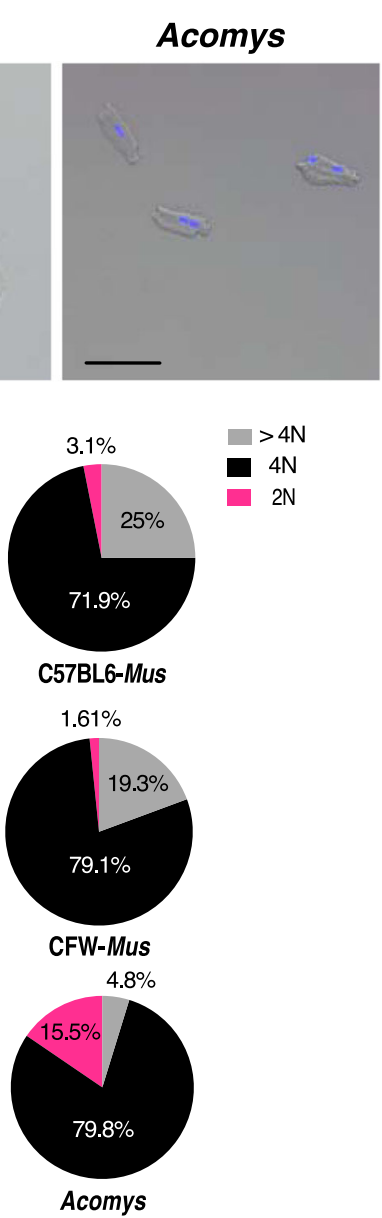

b

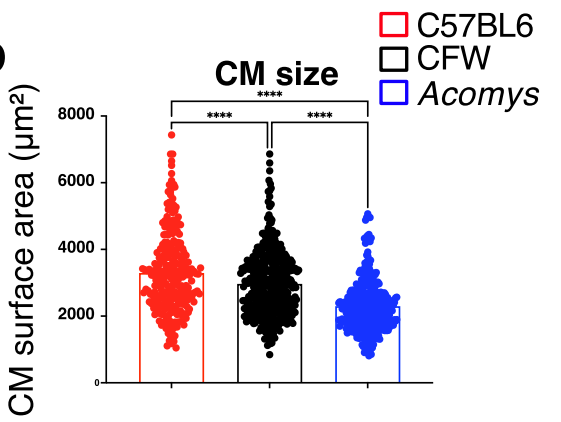

CM Nucleation

C

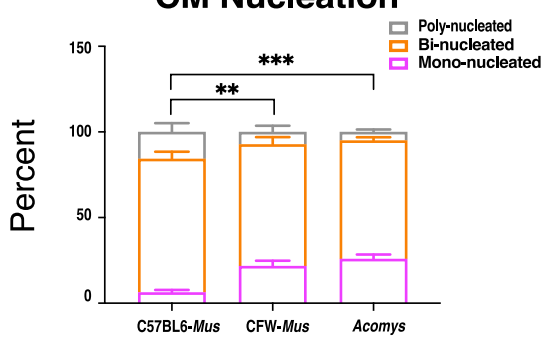

d

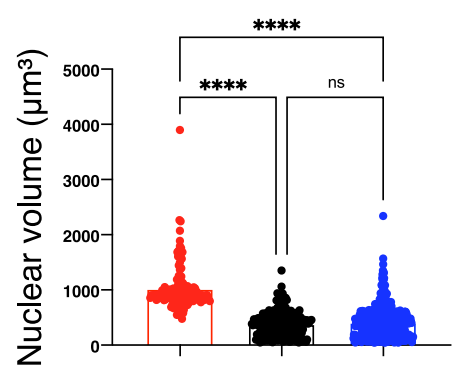

Fig. 2 Acomys cardiomyocytes exhibit features associated with Mus immature cardiomyocytes. a Representative images of single-cell ventricular suspension stained with DAPI (blue), identifying small, mononuclear, and binucleated cardiomyocytes in Acomys and larger binucleated cardiomyocytes in Mus-C57BL6 with Mus-CFW cardiomyocytes being intermediate in size (scale bar = 50 $\mu \mathrm{m}$ ) with mononuclear and binucleated cardiomyocytes. b Quantitative analyses of cell surface area of Mus and Acomys $(n=80-120$ randomly selected CMs/animal and $N=4$ each for Mus-C57BL6, Mus-CFW, and Acomys, ${ }^{* *} P<0.001$ by one-way ANOVA; values are means \pm S.E.M). c Percentage of monobi- and poly-nuclear cardiomyocytes in C57BL6-, CFW-Mus and Acomys ( $n=80-120$ randomly selected CMs/animal, $N=4$ mice for each group, values are means \pm S.E.M, ${ }^{* * P}<0.01,{ }^{* * *} P<0.001$ by One-way ANOVA and Dunnett's post-hoc analysis, compared to C57BL6-Mus). d Nuclear volume among isolated cardiomyocytes showing small nuclear volume in CFW-Mus and Acomys compared to C57BL6-Mus ( $n=80-120$ randomly selected CMs/animal, $N=4$ mice per group, values are means \pm S.E.M, ${ }^{* * *} P<0.001$ by One-way ANOVA and Dunnett's post-hoc analysis, compared to C57BL6-Mus). e Violin plots, on left, demonstrating the distribution of DAPI fluorescence intensity in cardiomyocyte nuclei as assessed using Imaris on 3D (Z-stack) microscopy images. The right panel shows the percentage of CMs within each ploidy category from the total pool of CMs from each species ( $2 \mathrm{~N}, 4 \mathrm{~N}$, and $>4 \mathrm{~N}) ; n=84-161$ randomly selected CMs/species).

coronary anatomy in all species. Relevant to permanent coronary ligation, all three demonstrated a similar course of the left anterior descending artery (LAD) supplying the anterior wall of the left ventricle (Fig. 1k). Coronary blood vessels adopt a hierarchical structure and vascular density leads to enhanced blood supply, angiogenesis, and ischemic resistance ${ }^{23,24}$. Thus, we analyzed the bifurcation points of the coronary tree as it supplies the anterior wall of the left ventricle. These analyses showed a similar density of branching points per major artery across species (Fig. 1l). Finally, we assessed capillary density at the mid left ventricular level between species and observed slightly higher capillary density in Acomys and CFW-Mus compared to C57BL6-Mus (Supplementary Fig. 2). While some of the baseline comparisons were statistically significant, these differences were not biologically relevant and did not correlate with post-injury response. These data demonstrated comparable cardiac anatomy and physiology between Acomys and the two Mus strains, C57BL6 and CFW.
Adult Acomys exhibit a high percentage of cardiomyocytes possessing characteristics associated with immature Mus ventricular cardiomyocytes

Ventricular CM phenotype varies across species and chronological age where it can influence the cardiac response to injury ${ }^{12,21,25,26}$. In mammals, a high percentage of mononuclear diploid CMs is observed in neonates and is indicative of an immature heart phenotype that is rarely seen in adult mammals ${ }^{27,28}$. To examine CM phenotype between species, we isolated ventricular CMs from Mus and Acomys and examined their size, number of nuclei/CM, nuclear characteristics, and ploidy (Fig. 2). Similar to a prior report ${ }^{21}$, Acomys CMs were significantly smaller than C57BL6 and were approximately four times more likely to be mononucleated (Acomys: $25.9 \pm 5.1 \%$ vs. CFW-Mus: $21.7 \pm 3 \%$ vs. C57BL6-Mus: $6.3 \pm$ $2.8 \%, P<0.001$; Fig. 2a-c). Additionally, when we quantified the average nuclear volume across all CMs from Acomys and Mus we found that CM nuclei were smaller in Acomys (Fig. 2d) ${ }^{29}$. We then assessed the ploidy of ventricular CMs across species using 3-dimensional confocal microscopy ${ }^{30}$ and found a significantly 

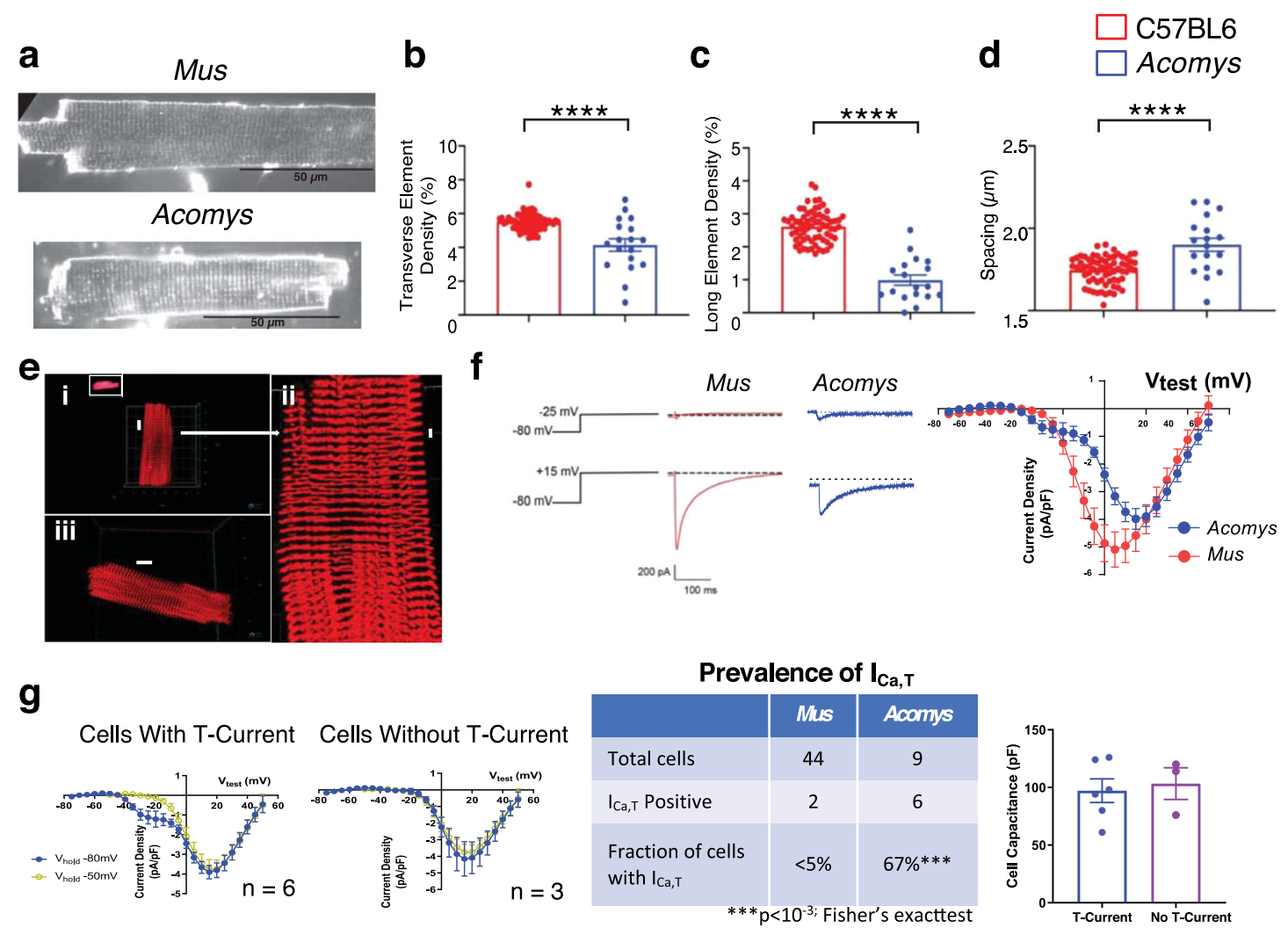

h

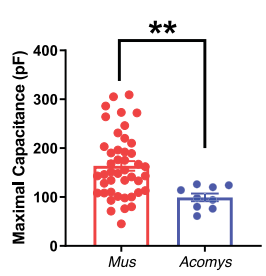

I
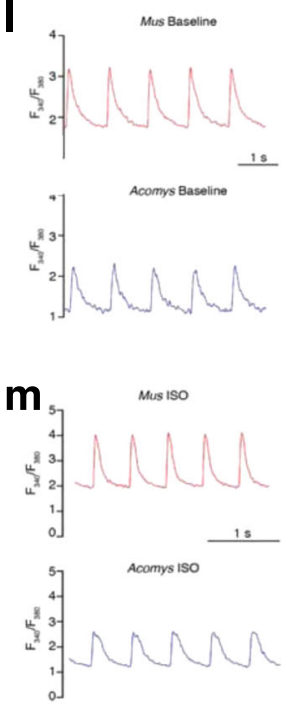

i

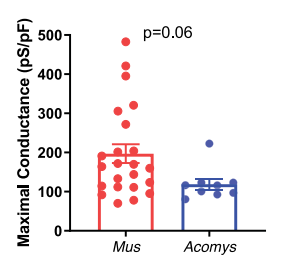

j

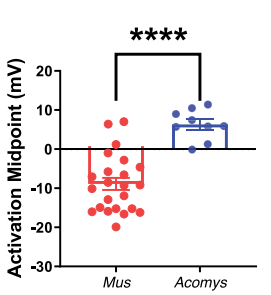

k

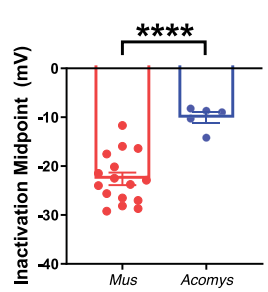

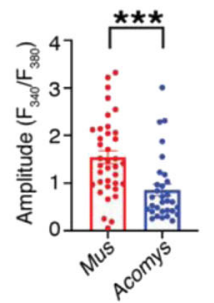

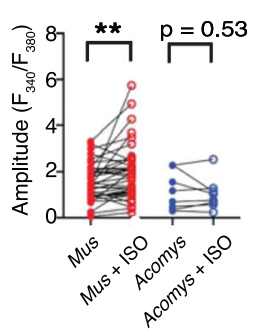

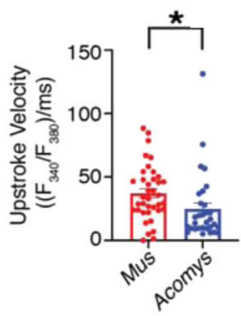
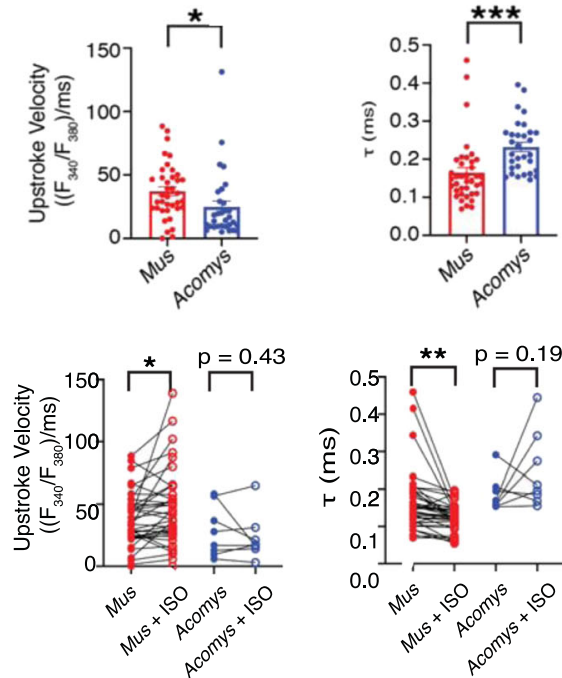

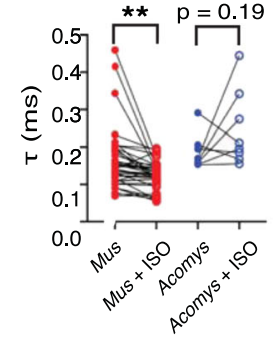

higher percentage of diploid CMs in Acomys compared to Mus with a corresponding lower percentage of $>4 \mathrm{~N}$ CMs (Fig. 2e). Compared to C57BL6 CMs, CFW-Mus CMs were smaller with a higher percentage of $\mathrm{CMs}$ being mononuclear, a finding that is similar to CM data collected for the inbred SWR/J strain ${ }^{25}$.
The relatively small cell size and higher mononucleation we observed in Acomys CMs suggested a higher percentage of CMs exhibiting characteristics observed in immature Mus CMs. T-tubule organization is highly regular in mature mouse $\mathrm{CMs}^{31}$. To assess T-tubule organization we stained CMs with di-8-ANEPPS (Fig. 3a) 
Fig. 3 Physiological characteristics of Acomys and Mus cardiomyocytes. a Representative images of isolated cardiomyocytes from C57BL6Mus and Acomys stained with di-8-ANEPPS to visualize T-tubules. T-tubule analysis shows significantly reduced T-tubule density in transverse (b) and axial directions (c) along with wider T-tubule spacing (d) in Acomys compared to Mus (C57BL6; $t=6.2,12$, and 4.4; $F=39,141$, and 19, for b-d, respectively; Mus $N=5, n=67$; Acomys $N=3, n=23$. e Acomys ventricular cardiomyocytes show organized z-discs. (i) Mononucleated $\mathrm{CM} \alpha$-actinin reveals highly organized z-disc structure (scale bar $=10 \mu \mathrm{m}$ ). (ii) expanded view of mononucleated $\mathrm{CM}$ in $\mathbf{i}(\mathrm{scale}$ bar $=2 \mu \mathrm{m}$ ). (iii) Representative binucleated CM (scale bars $=10 \mu \mathrm{m}$ ). f Current traces (left) current-voltage relationship (right) elicited by $V_{\text {test }}-25 \mathrm{mV}$ (left, upper) and $V_{\text {test }}+15 \mathrm{mV}$ (left, lower) from $V_{\text {hold }}-80 \mathrm{mV}$. g (Left) Current-voltage curves from $V_{\text {hold }}-80 \mathrm{mV}$ and $-50 \mathrm{mV}$ superimposed for Acomys CM exhibiting $\mathrm{I}_{\mathrm{Ca}, \mathrm{T}}(\mathrm{A})$ or no $\mathrm{I}_{\mathrm{Ca}, \mathrm{T}}$. $\mathbf{g}$ (Left center) $\mathrm{I}_{\mathrm{Ca}, \mathrm{T}}$ expression was heterogeneous but more Acomys ventricular CMs showed $\mathrm{I}_{\mathrm{Ca}, \mathrm{T}}$ compared to rare occurrences in Mus-C57BL6 (g, right center). $\mathbf{g}$ (Right) Cell capacitance was not different for Acomys CM with or without I $\mathrm{Ca}_{\mathrm{a}, \mathrm{T}}$ h Cell capacitance was greater in Mus-C57BL6 compared to Acomys $\left(t=2.88, F=7.9,{ }^{*} P<0.01\right)$. i Maximal conductance density trended greater in Mus-C57BL6 compared to Acomys $(t=2.0, F=7.7, P=0.06)$. j-k Voltage-dependent activation and inactivation of $\mathrm{I}_{\mathrm{Ca}, \mathrm{L}}$ was significantly shifted positive for Acomys compared to Mus-C57BL6 ( $t=5.8$ and $5.2, F=3.7$ and 4.5 , for $\mathbf{i}$, $\mathbf{j}$, respectively, ****P<0.001). I Representative calcium transients from isolated ventricular cardiomyocytes loaded with fura2-AM, Mus-C57BL6 (top, red) and Acomys (bottom, blue) paced at $1 \mathrm{~Hz}$. Scale bar: $2 \mathrm{~s}$. I (Left) Amplitude of the transients $(t=3.7, F=1.4, * * * P=0.0004)$. I (Middle) Velocity at which calcium enters the cytosol (upstroke of the transient: $\left.t=2.1, F=1.5,{ }^{*} P=0.04\right)$. I (Right) $(\tau)$ Calcium transient decay $(t=3.6, F=1.5, * * * P=$ 0.0005). $\mathbf{m}$ Representative calcium transients treated with $100 \mathrm{nM}$ isoproterenol (ISO), Mus-C57BL6 (top, red) and Acomys (bottom, blue), paced at $1 \mathrm{~Hz}$. Scale bar: $2 \mathrm{~s}$. (Left) Before and after ISO Amplitude of the transients $(t=2.8$ for Mus, $P=0.53, t=0.7$ for $A$ comys, $* * P=0.007$ ). (Middle) Before and after ISO Velocity at which calcium enters the cytosol $(t=2.5, P=0.02)$. (Right) Calcium transient decay $\left(t=3.4\right.$ for $M u s$, ${ }^{* *} P=0.002$; $N=7$ animals, $n=37$ cells for C57BL6-Mus; and $N=4$ animals, $n=31$ cells for Acomys).

and evaluated transverse and axial density along with T-tubule spacing. Acomys showed significantly reduced transverse and long element densities (Fig. 3b, c) with increased resting T-tubule spacing (Fig. 3d). In contrast to less regular T-tubule organization, Acomys showed highly registered z-discs (Fig. 3e and Supplementary Movie 1). To further explore the physiological implications of these findings, we conducted electrophysiological studies on isolated ventricular CMs from C57BL6-Mus and Acomys. In mammalian hearts, the expression of low-voltage activated ( $T$ type calcium channels) is an index of the fetal gene program in the ventricle; ${ }^{32}$ therefore, we recorded voltage-dependent $\mathrm{I}_{\mathrm{Ca}}$ from Acomys and Mus ventricular myocytes. From a $\mathrm{V}_{\text {hold }}-80 \mathrm{mV}$, a $V_{\text {test }}$ step to $-25 \mathrm{mV}$ elicited little if any discernible current in Mus (C57BL6) whereas, at $\mathrm{V}_{\text {test }}-25 \mathrm{mV}$, Acomys CMs exhibited a transient 'T-type' calcium current $\left(\mathrm{I}_{\mathrm{Ca}, \mathrm{T}} \mathrm{T}\right.$ (Fig. 3f, upper panel). Longer-lasting calcium current $\left(\mathrm{I}_{\mathrm{Ca}, \mathrm{L}}\right)$ was prominent with larger depolarizations in Mus (C57BL6) and Acomys (Fig. 3f, lower panel). The current-voltage relationship showed a prominent $\mathrm{I}_{\mathrm{Ca}, \mathrm{T}}$ component with a peak current between $\mathrm{V}_{\text {test }}-20$ and $-25 \mathrm{mV}$ in Acomys, but not in Mus (C57BL6) (Fig. 3f).

To further dissect $\mathrm{I}_{\mathrm{Ca}, \mathrm{T}}$ from $\mathrm{I}_{\mathrm{Ca}, \mathrm{L}}$, we performed current-voltage curve protocols from $V_{\text {hold }}-50 \mathrm{mV}$ to voltage-inactivate the $\mathrm{I}_{\mathrm{Ca}, \mathrm{T}}$ component (Fig. 3g). Current-voltage relationships for $\mathrm{I}_{\mathrm{Ca}, \mathrm{T}^{-}}$ expressing Acomys cells showed the prominent $\mathrm{I}_{\mathrm{C}, \mathrm{T}}$ appearing as a low-voltage activated current for $\mathrm{V}_{\text {hold }}-80 \mathrm{mV}$ but not from $\mathrm{V}_{\text {hold }}-50 \mathrm{mV}$ (Fig. 3g, left). 33\% of Acomys ventricular CMs showed no $I_{\mathrm{Ca}, \mathrm{T}}$ (Fig. $3 \mathrm{~g}$, center) but there was no observed correlation of cell size to presence of $\mathrm{I}_{\mathrm{Ca}, \mathrm{T}}$ (Fig. $3 \mathrm{~g}$, right). Cell capacitance was significantly lower in Acomys compared to Mus (C57BL6) (Fig. 3h), consistent with our morphometric analysis (Fig. 2b). Additionally, maximal conductance density trended greater in Mus (C57BL6) than Acomys (Fig. 3i). Voltage-dependent activation and inactivation of $\mathrm{I}_{\mathrm{Ca}, \mathrm{L}}$ was significantly shifted positive for Acomys compared to Mus (C57BL6) (Fig. 3j, k).

We next explored cytosolic calcium handling. Cytosolic $\mathrm{Ca}^{2+}$ transients (CaT) had a larger amplitude (Fig. 3l, left), faster upstroke (Fig. 3L, middle), and more rapid decay (Fig. 3l, right) in Mus (C57BL6) compared to that observed in Acomys. As with CM morphometrics (Fig. 2b-d) and the prevalent expression of a T-type calcium current (Fig. 3f, g) the smaller, slower CaT segregated with a less mature CM phenotype. Immature ventricular $\mathrm{CMs}$ also tend to show reduced $\beta$-adrenergic receptor $(\beta-A R)$ acute responsiveness ${ }^{33}$. Therefore, we tested the effect of isoproterenol challenge (ISO). To assess acute ISO responsiveness, we compared within-cell before and after ISO. Mus (C57BL6) showed increased amplitude and more rapid kinetics (Fig. $3 \mathrm{~m}$ ). By contrast, Acomys CaT amplitude and upstroke velocity were not significantly different $(P>0.05) . \mathrm{Ca}^{2+}$ re-uptake was accelerated by ISO in Mus but not in Acomys (Fig. 3m, right). Taken together, these data are consistent with an Acomys CM phenotype distinct from that of C57BL6-Mus. Moreover, these data demonstrate that a high percentage of Acomys CMs exhibit characteristics usually associated with Mus neonatal CMs.

\section{Acomys hearts demonstrate enhanced cardiac preservation after ischemic injury}

Acomys can regenerate complex tissue wounds, but the response to myocardial ischemic (MI) injury has not been extensively explored. Thus, we sought to compare the cardiac injury response between Acomys and the two strains of Mus using the permanent LAD-ligation model of $\mathrm{Ml}^{34-36}$. After injury, all groups showed a comparable drop in cardiac function, reflecting similar injury response as assessed by serial echocardiography (Fig. 4, Supplementary Fig. 3 and Supplementary Table 1). Indeed, there was no significant difference in most cardiac functional parameters $48 \mathrm{~h}$ after MI (Fig. 4b-f, $P>0.05$ ). When we assessed the extent of cardiac tissue injury in a subset of animals 3 days after MI using Masson's Trichrome and cardiac troponin T staining, we observed a comparable degree of $\mathrm{CM}$ loss and tissue injury between Acomys and the C57-Mus mice (Supplementary Fig. 3a, b). Furthermore, our cardiac MRI imaging showed comparable injury and involvement of the distal anterior wall and apex 3 days after MI between species (Supplementary Movies 2-5) as demonstrated by a similar area of late gadolinium enhancement (Supplementary Figs. 4 and 5). Our long-term echocardiography analyses showed stabilization in cardiac function and remodeling parameters in Acomys compared to the progressive deterioration seen in Mus strains (C57BL6 and CFW) up to 50 days after injury (Fig. 4b-f). In these analyses, we utilized the delta change from baseline to account for any differences in baseline function between species which allowed us to isolate the analyses to the changes occurring after MI. We observed consistent changes in left ventricular global ejection fraction, as well as measures of left ventricular adverse remodeling such as end-systolic and end-diastolic diameters and volumes (Fig. 4a-f and Supplementary Fig. 3c-g). The enhanced cardiac preservation and reduced adverse cardiac remodeling in Acomys was reflected by lower heart/BW ratio throughout the follow-up period extending to 50 days after MI (Fig. $4 \mathrm{~g}$ ), as well as the aforementioned echocardiographic evidence of smaller ventricular diameters. We also observed significantly lower wetdry lung weight in Acomys compared to Mus which is a measure of pulmonary edema and HF (Fig. 4h). Importantly, enhanced myocardial preservation in Acomys compared to Mus was 


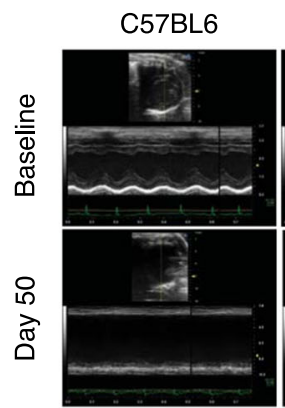

\section{b}

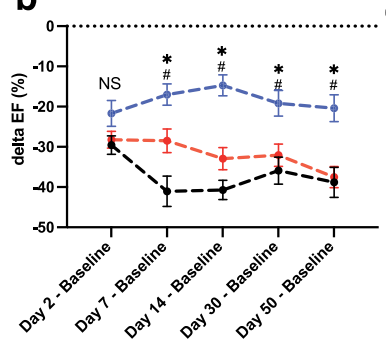

C
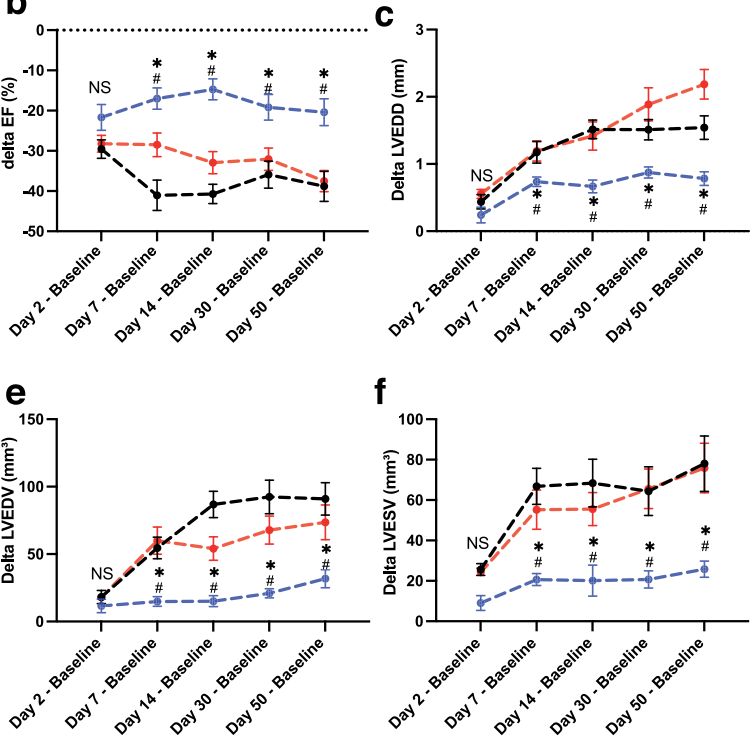

f

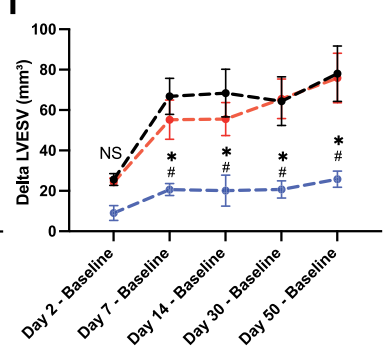

9
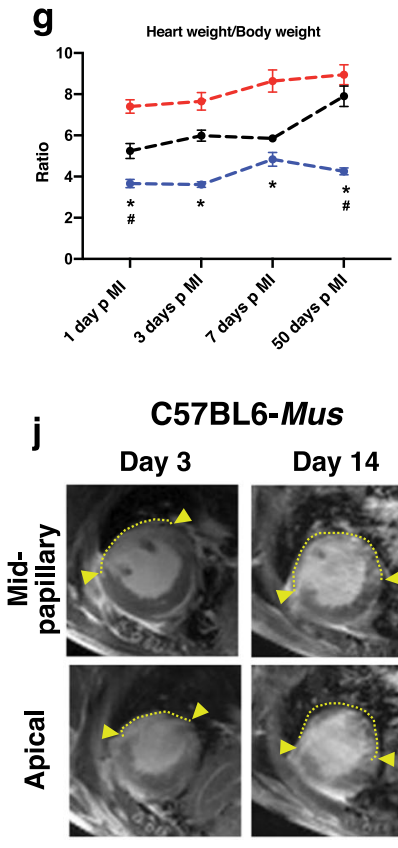

C57BL6-Mus

Day 14

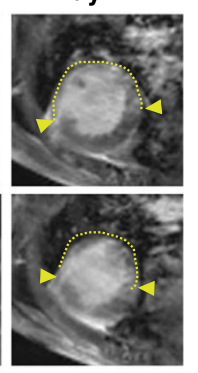

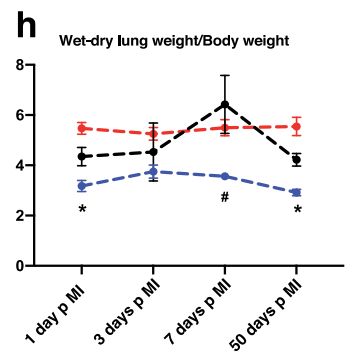
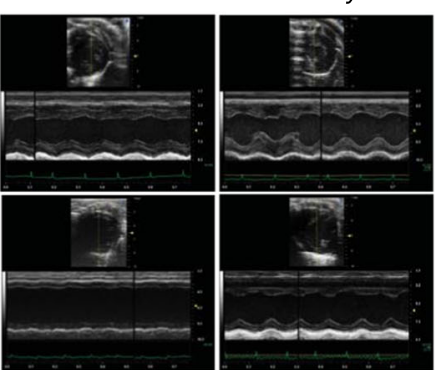

$\because$ C57BL6

d

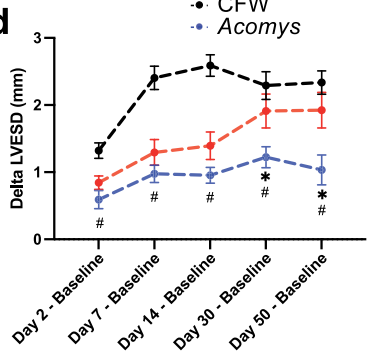

i

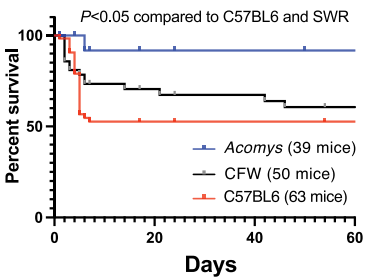

Acomys Day 3
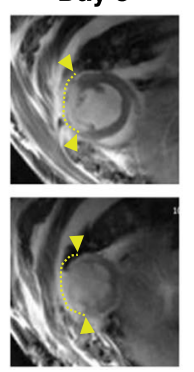

Day 14

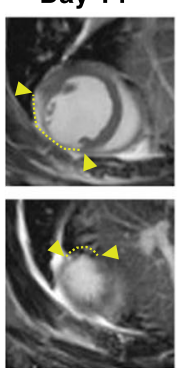

- C57BL6

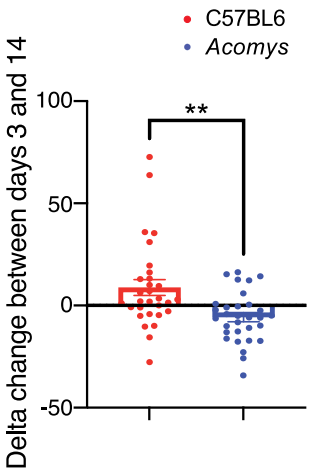

associated with a significant survival advantage (Fig. 4i). As seen in prior reports, the majority of mortality across all species was observed in the first week after MI. While we observed cardiac rupture in 17 C57BL6 and 14 CFW mice, we observed none in Acomys as visualized in post-mortem necropsies.
CMR studies demonstrated reduced scar progression between day 3 (D3) and D14 in Acomys compared to the large expanding infarct seen in Mus (Fig. 4j). Our long-term follow-up studies reflected significantly smaller infarct size in Acomys compared to Mus (C57BL6 and CFW) when assessed using Masson's Trichrome 
Fig. 4 Myocardial ischemic injury response in Acomys and Mus. a Representative M-mode echocardiography images of Mus-C57BL6 (left), Mus-CFW (middle), and Acomys (right) at baseline (upper images) and day 50 after MI (lower images). Delta ( $\Delta$ ) ejection fraction (EF) (b), $\Delta$ left ventricular end-diastolic diameter (LVEDD) (c), $\Delta$ left ventricular end-systolic diameter (LVESD) (d), $\Delta$ left ventricular end-diastolic volume (LVEDV) (e), and $\Delta$ left ventricular end-systolic volume (LVESV) (f) after injury, calculated as difference between each time point and baseline value in the same animal ( $N=16-45$ Mus-C57BL6, 10-20 Mus-CFW and 8-11 Acomys) (Values are means \pm S.E.M, ${ }^{*} P<0.05$ by Mixed-effects ANOVA, compared to *Mus-C57BL6 or "Mus-CFW). g Quantitation of heart weight (HW) normalized by body weight (BW) for Mus-C57BL6 ( $N=$ 2-26 animals), Mus-CFW ( $N=3-11$ animals) and Acomys $(N=2-13$ animals) at baseline and various timepoints after Ml suggesting maintenance of HW/BW ratio in Acomys and progressive increase in Mus (Values are means \pm S.E.M, $P<0.05$ by two-way independent ANOVA, compared to *Mus-C57BL6 or ${ }^{*}$ Mus-CFW). h Quantitation of wet-dry lung weight normalized by body weight of Mus-C57BL6 (2-26 animals), Mus-CFW (3-11 animals) and Acomys (1-13 animals) at various timepoints after Ml suggesting maintenance of wet-dry lung weight/body weight ratio in Acomys and progressive increase in Mus (Values are means \pm S.E.M, ${ }^{*} P<0.05$ by two-way independent ANOVA, compared to *Mus-C57BL6 or "Mus-CFW). i Kaplan-Meier analysis depicting mortality after MI and showing significantly lower mortality in Acomys compared with Mus (Gehan-Bareslow-Willcoxon test, $P<0.05)$. j Representative cardiac magnetic resonance (CMR) images showing comparably medium-sized infarct area in both species at 3 days post MI (arrows show the boundaries of late gadolinium enhancement/injury). The progression of infarct area was significantly slower in Acomys compared to Mus at 14 days after MI $(N=9-10$ animals per group, $t=3.28$, ${ }^{*} P<0.05$ by paired $T$-test).

staining 50 days after MI (Fig. 5a). We also saw similar results in our studies quantifying fibrosis using Picrosirius red staining 50 days after MI (Supplementary Fig. 3h). Using these Picrosirius stained sections to further explore the fibrous tissue alignment of the thicker scar in Acomys, we found that while the fiber alignment in the Mus strains was typical of a scar with highly parallel and compressed collagen bundles, fiber organization in the center of the Acomys scar was more porous with less compression (Fig. 5b). Furthermore, the resultant scar in Acomys was thicker across the entire scar which had a significantly higher cellular density (Fig. 5c), a finding that could potentially explain the lower rupture and mortality rates (Fig. 4i). Moreover, our serial scar analysis at D3 (Supplementary Fig 3a), 7, 17, and 50 (Fig. 6a-d) after MI demonstrated a unique evolution in infarct size between Mus and Acomys which corroborated the CMR studies (Fig. 4j and Supplementary Figs. 4 and 5). Taken together, our results demonstrate that Acomys exhibit ischemic tolerance and enhanced myocardial preservation after ischemic injury compared to the inbred C57BL6- and outbred CFW-Mus strains, respectively.

\section{Acomys exhibit rapid and enhanced vascularization of the infarct region after ischemic injury}

Blood vessel formation following ischemic injury is essential for functional stabilization. Following ischemic injury, vascular damage ensues in the area surrounding the original infarct region and leads to infarct expansion, development of adverse cardiac remodeling and $\mathrm{HF}^{2,37-39}$. Several studies have shown successful reduction of infarct expansion through effective angiogenesis and vascular maturation ${ }^{40,41}$. We assessed the vascular density in the peri-infarct region in Acomys and Mus strains and observed an increased density of capillaries (as assessed by CD31 staining) in Acomys compared to Mus (Fig. 7a). As commonly observed in adult mammals following $\mathrm{Ml}$, both Mus strains exhibited reduced vascular density in the scar center; by contrast, Acomys showed enhanced vascular density extending into the scar center (Fig. 7a). Additionally, enhanced angiogenesis was linked to more proliferating endothelial cells (isolectin CD31+/EdU+) in the peri-infarct region and scar center in Acomys compared to both Mus strains (Fig. 7b). We confirmed these findings using isolectin B4 staining for endothelial cells. These analyses corroborated our CD31 results demonstrating higher capillary density in the center of the scar and the higher proliferative nature of endothelial cells (Supplementary Fig. 6). In support of enhanced angiogenesis, we observed a significantly higher density of mature blood vessels $(\mathrm{a}-\mathrm{SMA}+)$ in the peri-infarct region in Acomys while the presence of these mature vessels was negligible in Mus strains (Fig. 7c). Taken together, in response to Ml our data support rapid and enhanced angiogenesis followed by vascular maturation in Acomys compared to Mus. This can explain, at least in part, the ischemic tolerance, reduced damage area and the thicker, cellularly dense scar in Acomys compared to Mus.

\section{DISCUSSION}

Spiny mice (Acomys) represent a unique mammalian model to explore endogenous cardiac repair consistent with their enhanced regenerative ability for a number of tissues and organs. In this study, we established the anatomical, functional, and cellular characteristics pre- and post-MI in Acomys compared to the inbred laboratory mouse strain C57BL6 and the outbred CFW strain. Our results demonstrated comparable cardiac structure, coronary anatomy, and functional parameters between the species allowing us to appropriately use these animal models for comparative heart injury studies. Interestingly, when comparing CMs across species we found that adult Acomys possessed a distinct CM phenotype that included features usually observed in immature or fetal Mus CMs such as small size, mononucleation, T-type calcium current, reduced t-tubule density and organization. After ischemic injury, Acomys exhibited enhanced ischemic tolerance and significant myocardial preservation, resulting in reduced adverse cardiac remodeling, smaller scar size and, importantly, better survival. Overall, our data support that Acomys show resistance to the type of cardiac damage that compromises other adult mammals, including humans, and supports future cardiac studies using this model to explore the mechanisms underlying their enhanced angiogenic response to myocardial infarct (MI).

Similar to other highly regenerative vertebrates, Acomys have emerged as a bona fide mammalian regeneration model for their ability to naturally regrow complex tissues following full-thickness skin injury ${ }^{14-19}$ and excision of musculoskeletal tissue from the ear pinna ${ }^{16,18,42}$. In each type of injury, adult Acomys were capable of restoring functional tissues, a phenotype that has not been observed in other adult rodents. Our gross anatomical studies established a similar coronary anatomy across species ${ }^{43}$ and we found that Acomys hearts were similar structurally and anatomically to Mus. Importantly, in vivo cardiac functional parameters were comparable between the three groups. These findings provided the appropriate foundation for the current study and lay the groundwork for follow-up studies that can inform new therapeutic strategies for ischemic heart disease.

Increased mortality and incidence of HF following $\mathrm{MI}$ remain prevalent in modern medicine. While advanced revascularization therapies and mechanical support have slightly improved survival in patients with Ml, millions develop progressive HF every year. Remarkably, Acomys exhibited significantly lower mortality after MI compared to both Mus strains and this reduction in mortality was related to a reduced incidence of cardiac rupture among Acomys. In fact, our histological assessment combined with cardiac MRI studies indicated significant differences in infarct evolution 
a

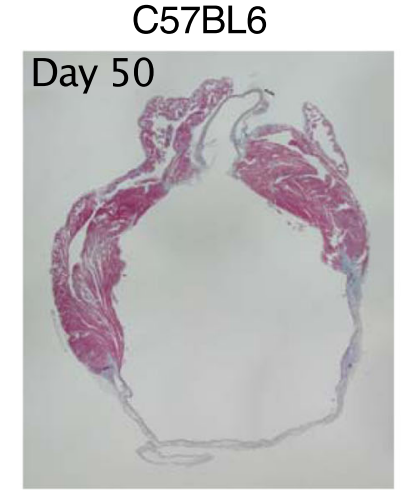

b
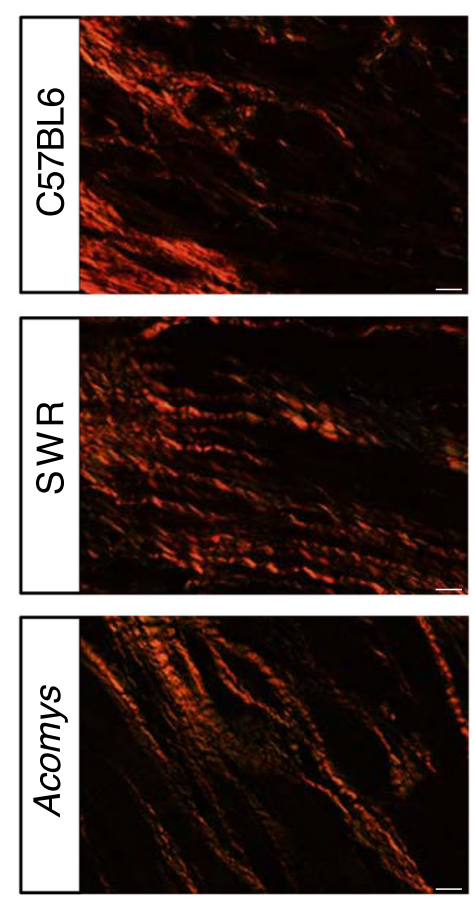

C

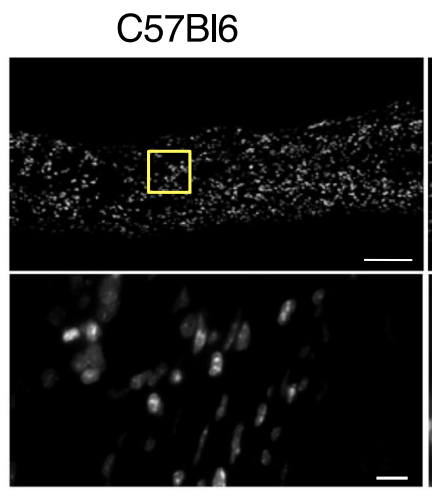

CFW

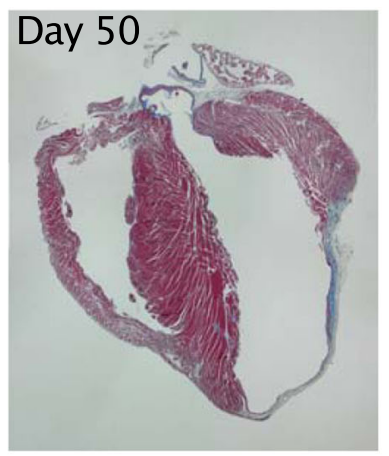

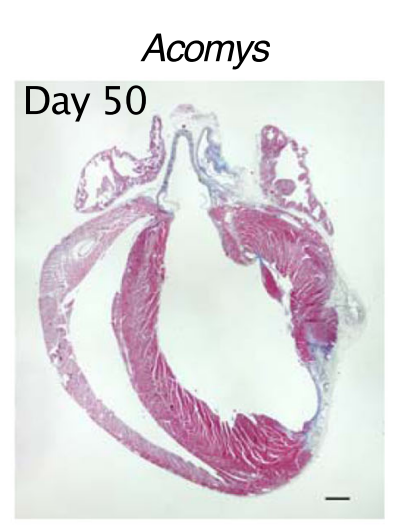

Scar
C57BL6

CFW

Acomys

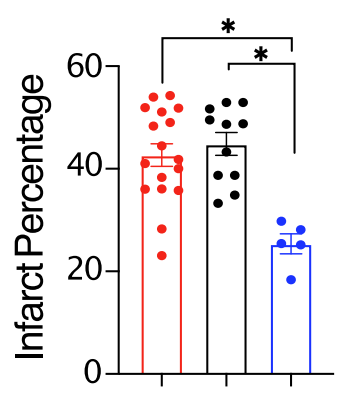

Day 50
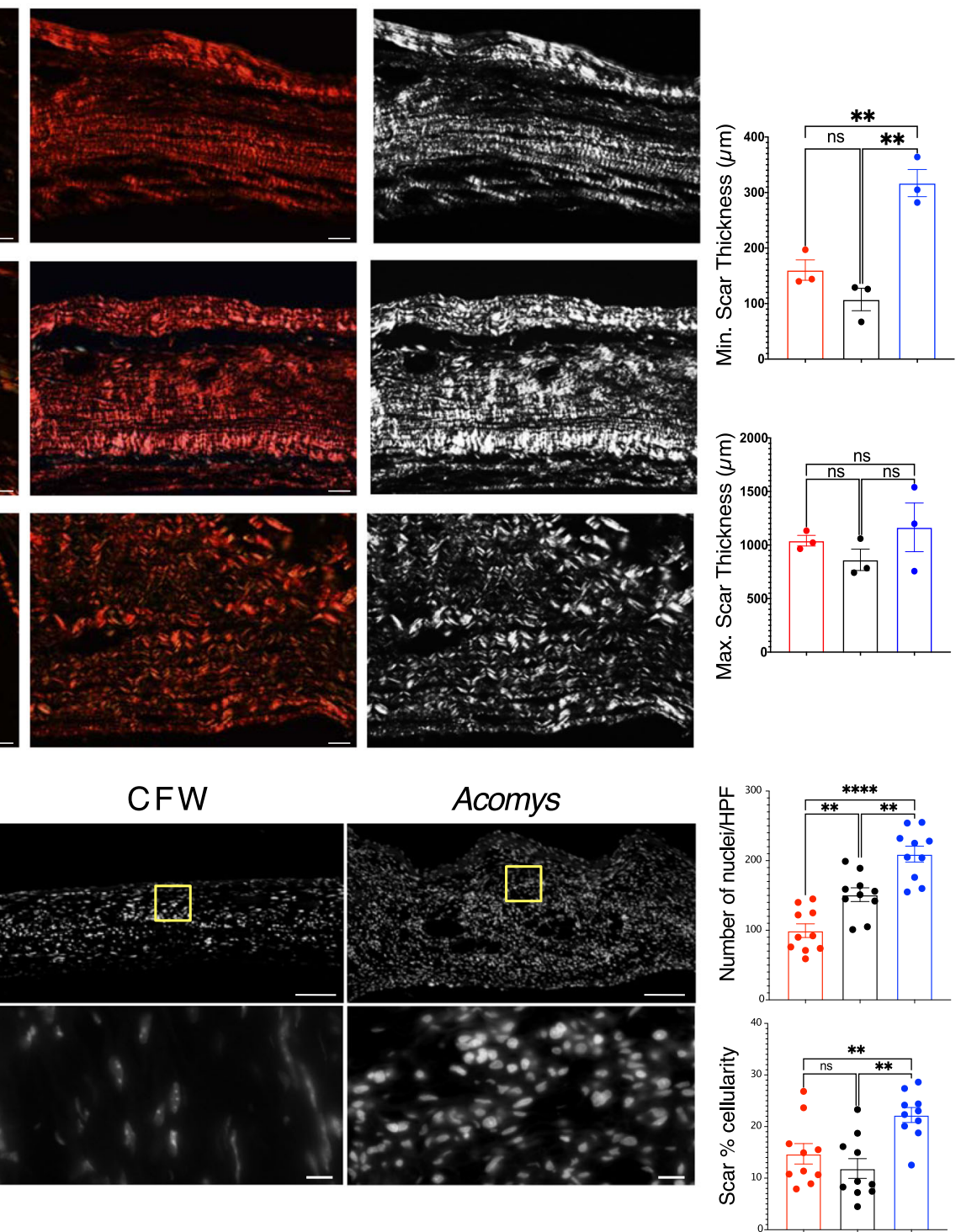

between species. While Mus demonstrated large infarct size, associated with thinning of the anterior wall, Acomys showed reduction in damage area despite starting with a comparable area of injury. These differences in infarct progression led to favorable cardiac remodeling and functional preservation demonstrated in our echocardiography studies.
This unique phenomenon was associated with a rapid angiogenic response that eventually led to superior ischemic tolerance. Acomys scars were thicker, more cellularized and had higher capillary and mature vessel density compared to Mus. This could at least partly explain the dramatically lower cardiac rupture rate and enhanced survival we observed in Acomys including the 
Fig. 5 Acomys exhibit cardiac tissue preservation in response to myocardial infarction. a Representative images of Masson's trichrome stained long-axis left ventricular cavity sections of Mus (C57BL6 and CFW) and Acomys 50 days post-MI (scale bar $=500 \mu \mathrm{m}$ ). Quantification of the infarct size, corresponding to the ratio between infarcted length and left ventricular length showing significantly smaller scar in Acomys compared to Mus strains ( $N=17$ Mus-C57BL6, 11 Mus-CFW, and 5 Acomys, values are means \pm S.E.M, ${ }^{*} P<0.05$ by one-way ANOVA and Tukey's multiple comparison test). b Representative images of picrosirius red stained scar center 50 days after Ml across species showing reduced alignment of collagen fibers in Acomys compared to Mus species. Both Mus strains show highly compressed, parallel collagen fibers, while Acomys fibers show more porosity between fibers and a wavy organization. Quantification of minimum and maximum scar thickness showing that Acomys has on average a comparatively thicker scar compared to Mus strains $(N=3$ animal/group, values are means \pm S.E.M, $* * P<0.01$ by one-way ANOVA and Tukey's multiple comparison test). There was no statistically significant difference in maximum scar thickness. c DAPI images were obtained in the center of the scar and demonstrate higher cellularity in Acomys compared to Mus strains (scale bar $=100 \mu \mathrm{m}$ in upper panel and $10 \mu \mathrm{m}$ in lower panel, yellow boxes indicating insets). Quantitative analysis showed higher density of nuclei and scar percentage cellularity in Acomys vs. Mus strains $\left(N=3\right.$ animal/group, values are means \pm S.E.M, ${ }^{* *} P<0.01$ by one-way ANOVA and Tukey's multiple comparison test).

stabilization of their cardiac function during long-term follow-up. A recent study found evidence that Acomys recovered many smaller coronary blood vessels 28 days after an LAD injury, evidence which supports our results and the utility of Acomys as a unique model of cardiac recovery ${ }^{22}$. While the data presented in this paper does not indicate cardiac regeneration to pre-injury levels, the dramatic ischemic tolerance and myocardial preservation noted in Acomys was associated with an enhanced angiogenic response. In fact, myocardial injury after $\mathrm{Ml}$ is related to tissue edema and microvascular obstruction and our CMR and IHC assessments show significant reduction in damage area between D3 and 7 in Acomys. These dynamic changes could be related to rapid resolution of tissue edema which is closely linked to enhanced angiogenesis. Indeed, preclinical and human MI studies have linked enhanced angiogenesis and vessel maturation with lower rates of infarct expansion ${ }^{41,44}$. While our data do not exclude the possibility that Acomys vascular cells are more resistant to ischemia, this presumed mechanism is less likely to explain the increased vascular density in the infarct region given the observed superior endothelial cell proliferation and vessel maturation noted in Acomys in our studies.

The mammalian heart loses its reparative capability after the first week of life where heart damage results in permanent loss of myocardium combined with fibrotic scarring and adverse remodeling ${ }^{12}$. The transition to fibrotic repair is closely linked with the loss of CM proliferation as CMs enter cell cycle arrest and become mononuclear polyploid or multinucleated ${ }^{12,45}$. However, in other vertebrate species where CMs maintain the lifelong ability to proliferate (e.g., zebrafish and newts), cardiac regeneration is still possible ${ }^{45,46}$. One of the hallmarks of regenerative hearts is the relatively high frequency of mononucleated diploid $\mathrm{CMs}^{45-48}$. This evidence suggests that higher proportions of mononuclear diploid CMs are associated with higher regenerative potential following ischemic injury ${ }^{25}$. Interestingly, our results showed that outbred CFW-Mus possessed an intermediate CM phenotype between Acomys and C57BL6 with respect to CM size and nuclei number. Despite possessing this CM phenotype, outbred CFW had nearly the same mortality as C57BL6 suggesting that rapid angiogenesis may be a key component to facilitate the protective features conferred by having a larger population of smaller, mononuclear CMs. Recent studies across a large number of mouse strains suggest additional CM characteristics, including their metabolic profile, might act as key elements in cardiac recovery after ischemic injury ${ }^{26}$. These characteristics were not fully explored in our study and thus may have contributed to the limited recovery seen in our CFW strain. On the other hand, cardiac tissue requires vascularization for supporting the high metabolic activity of $\mathrm{CMs}^{37}$, and our current findings support rapid angiogenic invasion into new fibrotic tissue as a mechanism to resist cardiac damage. Future studies are necessary to explore CM behavior across species and how this may relate to enhanced angiogenesis.

There are two types of $\mathrm{Ca}^{2+}$ channels in CMs, L-type, and T-type. L-type $\mathrm{Ca}^{2+}$ channels are highly expressed in the adult heart and are important therapeutic targets for the management of various cardiovascular diseases. In contrast, T-type $\mathrm{Ca}^{2+}$ channels are rarely found in adults and are present in fetal and early postnatal mice ${ }^{49}$. In our cell electrophysiology studies, adult Acomys exhibited a higher percentage of T-type $\mathrm{Ca}^{2+}$ channels. This feature is consistent with a higher prevalence of phenotypically 'young' CMs in Acomys compared to Mus (C57BL6). Furthermore, ventricular $\mathrm{CMs}$ in adult Acomys showed reduced $\mathrm{Ca}^{2+}$ transient amplitude, slower kinetics, and attenuated $\beta$-AR responsiveness. While this unique phenomenon in Acomys was associated with superior myocardial preservation after ischemic injury, because we did not examine $\mathrm{Ca}^{2+}$ channels in outbred CFW mice, we do not know if this feature is associated with the prevalence of small, diploid, mononucleated CMs. However, it is interesting to note that although our CM characterization studies showed that outbred CFW mice possessed a higher prevalence of small, diploid, mononucleated CMs compared to those from the C57BL6 strain, this feature alone did not confer the type of enhanced myocardial preservation response observed in previous work using inbred SWR/J mice ${ }^{25}$. This would suggest a synergy between CM phenotype and enhanced angiogenesis with respect to cardiac preservation following MI. Future studies are necessary to examine the proliferative potential of adult CMs in Acomys and Mus as well as their potential to adequately respond to myocardial injury.

In conclusion, our study presents Acomys as a unique model for cardiac research with comparable cardiac structure, function, and anatomy to C57BL6- and CFW-Mus. Acomys exhibited a distinct CM phenotype and angiogenic response resulting in enhanced recovery and ischemic tolerance after ischemic injury. It is important to note that while we do not see clear evidence of cardiac regeneration, the enhanced myocardial preservation seen in Acomys could uncover important therapeutic targets for millions of patients who develop ischemic cardiomyopathy after MI. Future studies will focus on the mechanism and potential for these phenotypic differences to foster myocardial preservation and repair instead of scarring and HF in models of cardiac disease.

\section{METHODS}

\section{Animal care}

Male Acomys cahirinus (6-8 months old, sexually mature animals) were obtained from our in-house breeding colony. Male Mus musculus (8-12 weeks, sexually mature animals) C57BL6/J (strain \#000664) were obtained from the Jackson Laboratory, Bar Harbor, ME, and outbred Swiss Webster (CFW) were obtained from Charles River (strain \# 024). Spiny mice were housed in their own building where they were maintained on an average 12:12 h L:D cycle with exposure to natural light and maintained on a diet of 14\% mouse chow (Teklad Global 2014, Harlan Laboratories) along with black sunflower seeds. Mus strains were maintained by the University of Kentucky, Division of Laboratory Animal Resources and were exposed to a strict 12:12 h L:D cycle and maintained on an 18\% mouse chow (Tekland Global 2918, Harlan Laboratories, Indianapolis, IN) diet. All animal procedures were approved by the University of Kentucky Institutional 

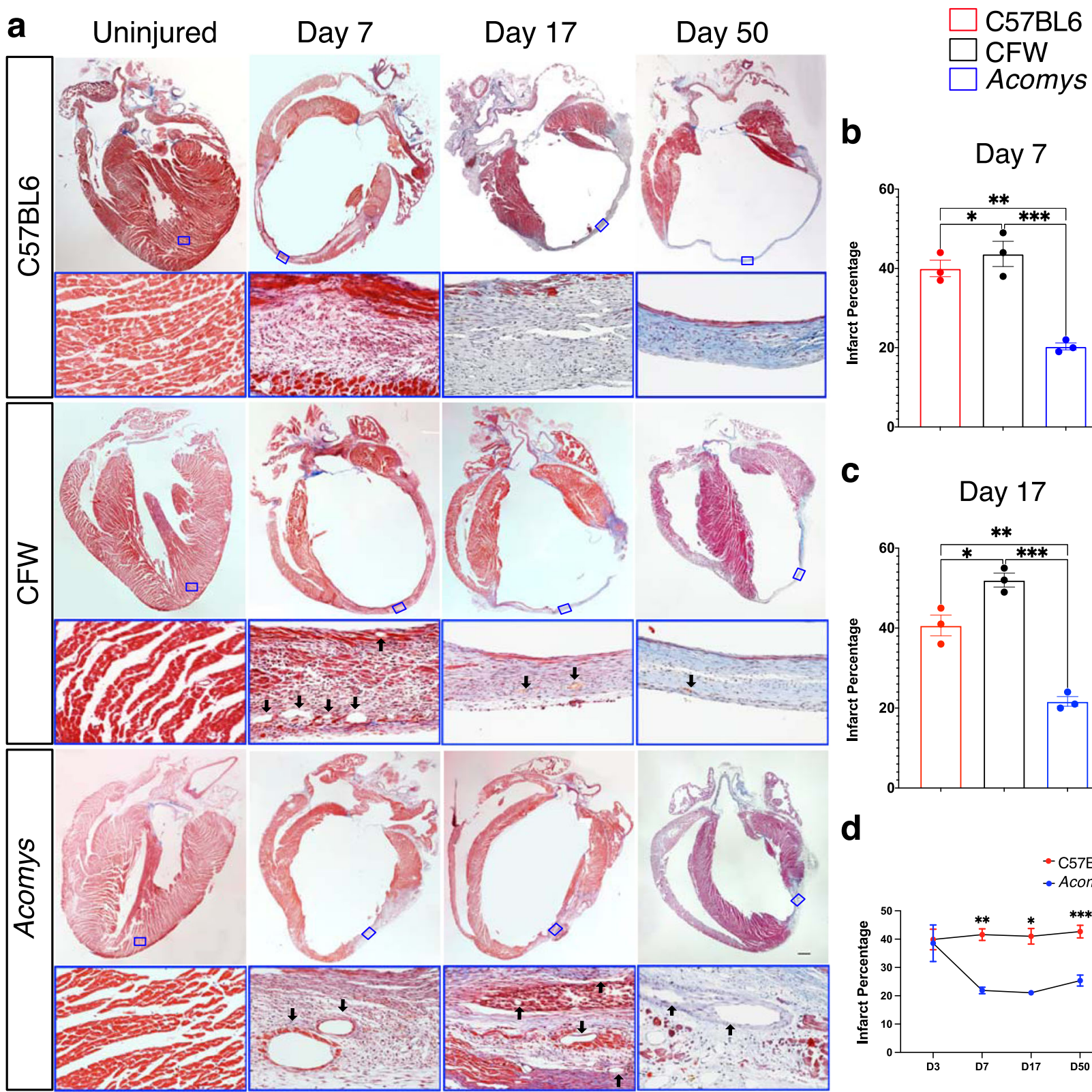

b

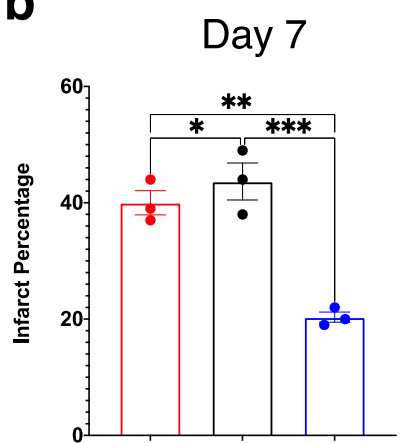

C

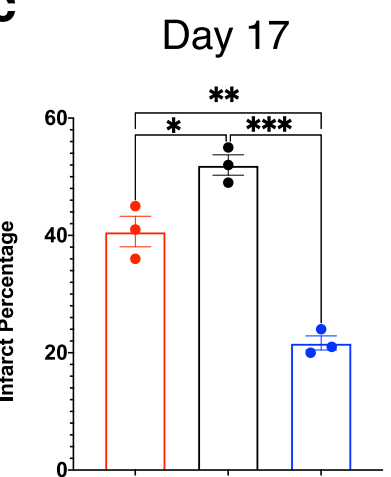

d

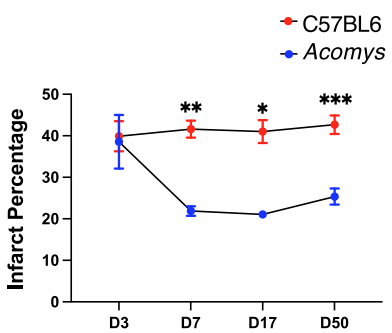

Fig. 6 Cardiac scar tissue exhibits limited expansion in Acomys. a Representative images of Masson's trichrome stained long-axis left ventricular cavity sections of Mus (C57BL6 and CFW) and Acomys in uninjured and MI animals at 7-, 17- and 50-days post-Ml showing the progression of scar across species (scale bar $=500 \mu \mathrm{m}$ ). The lower panels represent the boxed areas and show the composition of the scar at each stage. b, c Quantification of the infarct size, corresponding to the ratio between infarcted length and left ventricular length showing significantly smaller scar in Acomys compared to Mus strains ( $N=17$ Mus-C57BL6, 11 Mus-CFW, and 5 Acomys, values are means \pm S.E.M, * $P<$ 0.05 by one-way ANOVA and Tukey's multiple comparison test). $\mathbf{d}$ Scar progression in Acomys and Mus-C57BL6 showing dynamic reduction in infarct size in Acomys in contrast to the larger static scar in Mus-C57BL6 $\left({ }^{*} P<0.05,{ }^{* *} P<0.01\right.$, and ${ }^{* * *} P<0.001$ as analyzed using 2 -way independent ANOVA).

Animal Care and Use Committee (IACUC \#: 2019-3254, 2013-1155, 20213739, and 2011-0889).

\section{Coronary anatomy}

Coronary anatomy was visualized using corrosion casting technique. Mice received an i.p. injection of heparin (100 units in $300 \mu \mathrm{l}$ sterile PBS) to prevent coagulation. Ten minutes later, under isoflurane anesthesia, hearts were excised and cannulated with a blunt tip $23 \mathrm{~g}$ needle and clamp. $10 \mathrm{ml}$ of $1 \times$ PBS was perfused via ascending aorta, followed by $1.5 \mathrm{ml}$ Batson's 17 polymer mixture $(2.5 \mathrm{ml}$ base monomer $+600 \mu \mathrm{l}$ Catalyst +3 drops Promoter; Polysciences). Polymer was mixed and 10 min later was injected over $5 \mathrm{~min}$ and then left to cure on ice for $3 \mathrm{~h}$. Once cured, hearts were briefly washed with $\mathrm{ddH} 2 \mathrm{O}$ and then placed in potassium hydroxide (maceration solution) for $1 \mathrm{~h}$ at room temp. After maceration incubation, hearts were laid with the anterior side up and the LAD artery was imaged using a Nikon Camera (DS-12) after application of several drops of ddH2O to clear overlying tissue. Images were quantified using Imaris ${ }^{\oplus}$ Filament Tracking software. First, the blue channel was extracted and changed to mono. A Gaussian filter was applied, background was subtracted, and then semi-automatic tracing was used to identify vessels and branching points. Branching points of all vessels that supply the left ventricular wall were quantified for each species. Five representative images for each species are shown in Fig. 1k, l. 
a

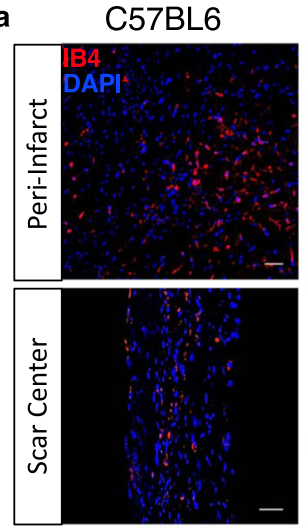

b
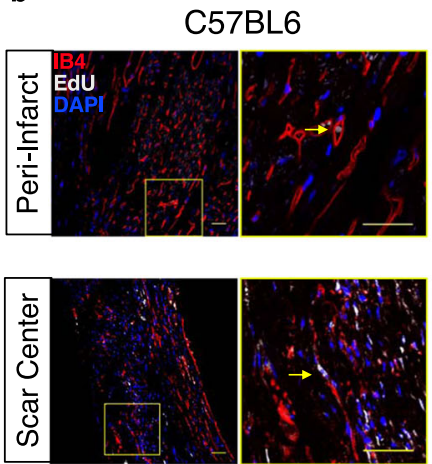

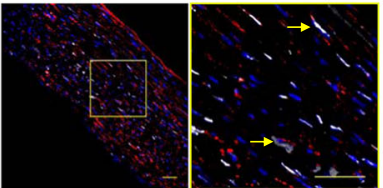

Acomys
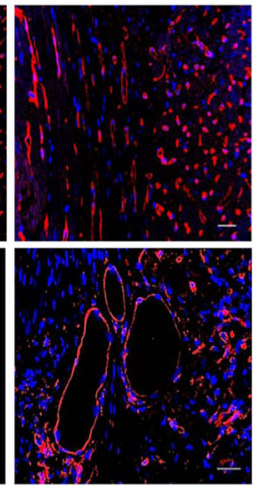

CFW
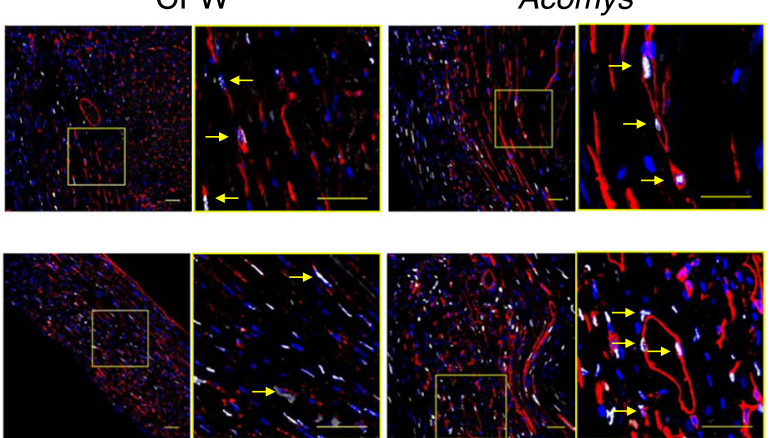

Infarct Area

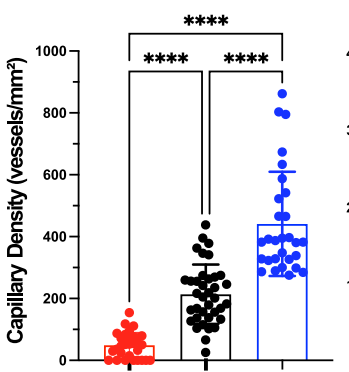

Acomys c
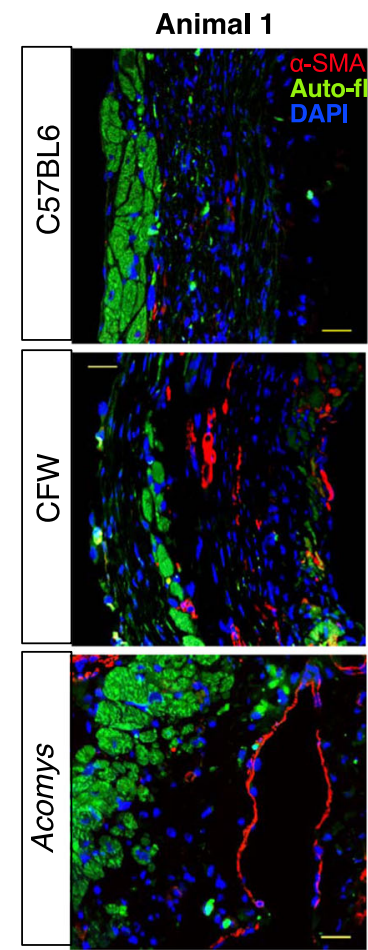

Animal 2
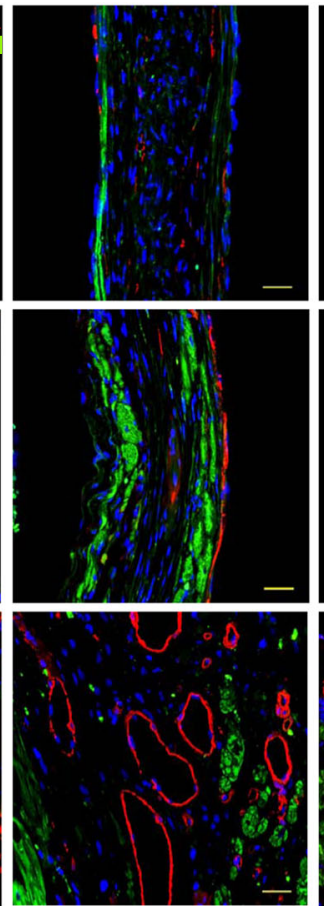

Animal 3
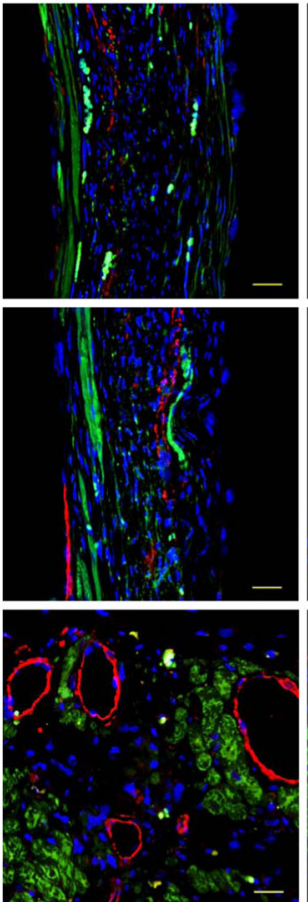

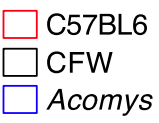

Peri-Infarct Area
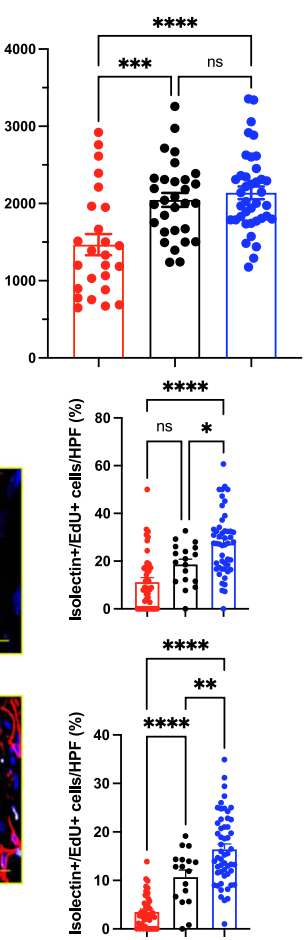

\section{EdU administration}

To assess angiogenesis, proliferating vessels were quantified in both the periinfarct and scar regions in all strains 17 days post-Ml. Mice received a single intraperitoneal injection of 5-ethynyl-2'-deoxyuridine (EdU, $10 \mathrm{mg} / \mathrm{kg}$, Cayman Chemical, Ann Arbor, MI) immediately after Ml surgery and continuing daily through post-op Day 14. Hearts were collected on Day 17 and processed as indicated in Histology and Immunofluorescence sections. Hearts were stained for Isolectin or CD31, and EdU with a DAPI counterstain. Images were taken with a $\times 40$ oil immersion objective on a Nikon A1 Confocal Microscope in the University of Kentucky Confocal Microscopy facility. 8-15 images per section were taken and the data reported as percentage of Isolectin + or CD31+ cells that also expressed EdU. 
Fig. 7 Enhanced angiogenesis and vessel maturation in the infarct region following MI in Acomys compared to Mus strains. a Representative images of CD31 staining (red) defining endothelial cells in the peri-infarct region and the center of the scar at the left ventricular cavity level of Mus (C57BL6 and CFW) and Acomys 50 days post-MI (scale bar $=25 \mu \mathrm{m}$ ). Quantification of capillary density showing significantly higher capillary density in Acomys compared to Mus-C57BL6 in the peri-infarct region and to both Mus strains in the center of the scar ( $N=4$ Mus-C57BL6, 4 Mus-CFW, and 5 Acomys, values are means \pm S.E.M, ${ }^{* * *} P<0.001$ and ${ }^{* * *} P<0.0001$ by one-way ANOVA and Tukey's multiple comparison test). b Representative images of CD31 (green, pseudocolored) and EdU staining (magenta) marking proliferating endothelial cells in the peri-infarct region and the center of the scar in Mus (C57BL6, left, and CFW, center) and Acomys (right) 17 days post-Ml (scale bar $=25 \mu \mathrm{m}$ ). Insets show higher magnification of vascular structures. Quantification of percentage of CD31+ cells expressing EdU, showing significantly higher endothelial cell proliferation in Acomys compared to the two Mus strains in both peri-infarct and scar center areas $(N=4$ Mus-C57BL6, 4 Mus-CFW, and 5 Acomys, values are means \pm S.E.M, **** $P<0.0001$ by one-way ANOVA and Tukey's multiple comparison test). c Representative images of smooth muscle actin staining (red) in the scar center region showing higher prevalence of medium-sized blood vessels in Acomys compared to Mus strains. Autofluorescence (green) was used to identify the infarct boundary (images shown from 4 animals/group, scale bar $=25 \mu \mathrm{m})$.

\section{Tissue collection and histology}

Tissue (heart and lung) from all species were collected and weighed immediately at sacrifice. The dry lung weight was collected after 3 days of $65^{\circ} \mathrm{C}$ incubation. Hearts were perfused with PBS (VWR International) followed by $4 \%$ PFA (VWR International) fixation via cannulation of the ascending aorta. Hearts were post-fixed overnight at $4{ }^{\circ} \mathrm{C}$. Hearts were then sectioned in half long axis at the level of the ligation and transferred to $70 \%$ ethanol until sectioning. Tissues were then paraffin embedded and sectioned. All tissue was sectioned at $5 \mu \mathrm{m}$ for immunofluorescence and $8 \mu \mathrm{m}$ for Masson's Trichrome and Picrosirius Red staining. Masson's Trichrome was imaged using an Olympus BX53 microscope (Olympus, Tokyo, Japan) at $\times 4$ and $\times 40$ to evaluate scar. The scar size was quantified by examining and calculating the average percent of fibrosis of the total LV area using NIH ImageJ 1.46R software based on Masson's trichrome staining. Picrosirius red staining was performed to visualize scar collagen organization at the peri-infarct and infarct regions. Images of picrosirius stained slides were captured at $\times 40$ magnification using Olympus BX53 microscope (Olympus, Tokyo, Japan). The minimum and maximum scar cross sectional length was measured using ImageJ to determine the thickness of the scar. DAPI counterstain on ventricular heart sections was performed to analyze cellular density of scar tissue. Images of DAPI stained tissue were taken at $\times 4$ and $\times 60$ magnification using Olympus BX53 microscope in the University of Kentucky Light Microscopy Core. The number of nuclei in each imaging field was counted using ImageJ. All measurements were analyzed by blinded observers.

\section{Immunofluorescence}

Immunofluorescence assessments were carried out on deparaffinized and rehydrated sections as previously described ${ }^{50}$. After deparaffinization, slides were washed, and TRIS (Citrate for cardiac troponin T) heat induced epitope retrieval was performed for $25 \mathrm{~min}$. For Isolectin (IB4)/EdU and CD31/EdU staining, the following EdU staining was performed between the antigen retrieval and protein blocking steps. A reaction cocktail was created using $5 \mu \mathrm{l} 2 \mathrm{M}$ Tris (pH8.5; Thermo), $2 \mu \mathrm{l} 50 \mathrm{mMCuSO}_{4}$ (Fisher), $20 \mu \mathrm{l}$ $0.5 \mathrm{M}$ fresh ascorbic acid (Thermo), $2 \mu \mathrm{l}$ Alexa Fluor Azide $(0.25 \mathrm{mg} / \mathrm{ml}$, Thermo) and $73 \mu \mathrm{lddH} 20$ per slide. Slides were incubated in the dark in reaction cocktail for $30 \mathrm{~min}$ at room temperature, then washed in PBS. Protein blocking (3\%) was performed for $30 \mathrm{~min}$ and slides were washed in PBS. Permeabilization was attained with $0.3 \%$ Triton X-100 for alpha smooth muscle actin, cardiac troponin T (cTNT) staining. IB4 stained slides were also blocked for streptavidin/biotin (SP-2002 Vector Labs) according to manufacturer's protocol. After blocking, slides were washed in PBS and incubated with primary antibody: Isolectin B4 (L-2140, Sigma, 1:500), CD31 (AF3628, R\&D Systems, 1:100), CTNT (MA5-12960, Invitrogen, 1:100) or alpha smooth muscle actin (a-SMA, MAB1420, R\&D Systems, 1:50) overnight at $4{ }^{\circ} \mathrm{C}$. After washing in $\mathrm{PBS}$, sections were incubated with secondary antibody: streptavidin-Alexa Fluor 568 (S11226, Thermo Fisher, 1:500) for IB4, Donkey anti goat Alexa Fluor 568 (ab175704, Abcam, 1:500) for CD31, or Donkey anti-mouse Alexa Fluor 568 (ab175700, Abcam, 1:500) for CTNT and a-SMA for $30 \mathrm{~min}$ at room temperature. Slides were washed and cover slipped with antifade mounting medium containing DAPI counterstain (H-1200, Vector Labs). 8-15 adjacent areas per section were examined at $\times 40$ magnification using Nikon A1 Confocal Microscope in the University of Kentucky Confocal Microscopy facility. IB4 staining was performed on tissues from day 50 post-Ml and was quantified at both the peri-infarct border and the center of the scar and is presented as total capillary density per $\mathrm{mm}^{2}$. Baseline IB4 measurements were taken at a comparable location to the peri-infarct area. To identify vessels in the periinfarct and infarct regions 50 days post $\mathrm{MI}, \mathrm{CD} 31$ staining was performed and the number of complete vessels with multiple nuclei and open lumen were quantified in 9-15 images per section per area in all species. To determine vessel proliferation at 17 days post-MI using either IB4/EdU or CD31/EdU, 8-15 images per section were taken and the data reported as percentage of IB4+ or CD31+ cells that also expressed EdU.

To identify extent of damage at 3 days post-MI, we stained for cTNT and Wheat Germ Agglutinin 488 (WGA488, L4895, Sigma, 1:50). In this case, WGA488 was incubated after blocking and before permeabilization steps listed above for $15 \mathrm{~min}$ at room temperature followed by 3 PBS washes. Images were taken on Zeiss Axioscanner for whole slice images, insets were obtained on Nikon AR1 confocal with a $\times 40$ oil immersion lens, both located in the University of Kentucky microscope core.

\section{Cardiomyocyte isolation}

Ventricular CMs were isolated as previously described. Briefly, animals received an i.p. injection of heparin $(0.3 \mathrm{ml}$ of 1000 units $/ \mathrm{ml})$ prior to sacrifice. Animals were then anesthetized with $1-3 \%$ Isoflurane. Hearts were excised and immediately perfused on a Langendorff apparatus with a high-potassium Tyrode buffer and then digested with 5-7 mg of liberase (Roche Applied Science). After digestion, atria were removed, and ventricular myocytes were mechanically dispersed. Some isolated ventricular CMs were used for measuring cell surface area and nuclei, and the others were used for electrophysiological recordings and calcium transients. For electrophysiology and calcium transient measurements control Mus were used containing Rad $\mathrm{f}^{\mathrm{f} / \mathrm{fl} 1}$. Some of this control Mus data appeared in Ahern et al. ${ }^{51}$. Calcium concentrations were gradually restored to physiological levels in a stepwise fashion for electrophysiological studies, and only healthy quiescent ventricular myocytes were used for electrophysiological analysis within $12 \mathrm{~h}$.

\section{Electrophysiological recordings and calcium transients}

$\mathrm{I}_{\mathrm{C}, \mathrm{L}}$ was recorded in the whole-cell configuration of the patch clamp technique as previously described ${ }^{51}$. All recordings were performed at room temperature $\left(20-22^{\circ} \mathrm{C}\right.$ ). The pipette solution consisted of (in mmol/ liter) $125 \mathrm{Cs}$-methanosulfonate, $15 \mathrm{TEA}-\mathrm{Cl}, 1 \mathrm{MgCl} 2,10 \mathrm{EGTA}$, and 5 Hepes, 5 MgATP, 5 phosphocreatine, $\mathrm{pH}$ 7.2. Bath solution contained (in mmol/ liter) $140 \mathrm{NaCl}, 5.4 \mathrm{KCl}, 1.2 \mathrm{KH} 2 \mathrm{PO} 4,5$ Hepes, 5.55 glucose, $1 \mathrm{MgCl} 2,1.8$ $\mathrm{CaCl} 2$, pH 7.4. Once a cell was successfully patched, zero sodium bath solution was introduced into the chamber (mmol/liter) $150 \mathrm{~N}$-methyl-Dglucamine, $2.5 \mathrm{CaCl} 2,1 \mathrm{MgCl} 2,10$ glucose, 10 Hepes, 4-amino-pyridine, $\mathrm{pH}$ 7.2. Recordings of ISO response were recorded in zero sodium bath solution containing $300 \mathrm{nM}$ ISO. $\mathrm{I}_{\mathrm{Ca}, \mathrm{L}}$ was recorded from a holding potential $\left(V_{\text {hold }}\right)$ of $-50 \mathrm{mV}$. $\mathrm{I}_{\mathrm{C}, \mathrm{T}}$ and $\mathrm{I}_{\mathrm{Ca}, \mathrm{L}}$ was recorded from $\mathrm{V}_{\text {hold }}-80 \mathrm{mV}$ with $300 \mathrm{~ms}$ depolarization steps to levels as shown in Fig. 3. Activation voltage dependence parameters were obtained by first transforming the peak current-voltage relationship to a conductance transform by fitting the ascending phase (typically $\mathrm{V}$ test +15 to $+40 \mathrm{mV}$ ) to a linear regression to obtain a reversal potential. Using $G=I / V$ the conductance as a function of voltage transform was then fitted to a Boltzmann distribution of the form $G$ $(V)=G m a x /\left[1+\exp \left(V_{1 / 2} / k\right)\right]$ where $G$ max is the maximal conductance and $V_{1 / 2}$ is the activation midpoint and $k$ is the slope factor. For the steady state availability curve, we pre-pulsed cells to Vpre ranging from -40 to $+10 \mathrm{mV}$ in $5 \mathrm{mV}$ increments and recorded a $V_{\text {test }}$ to $0 \mathrm{mV}$. Peak currents were 
plotted as a function of Vpre, and a Boltzmann distribution was fitted to the resulting curve.

Calcium transients were recorded from ventricular CMs loaded with cell permeable Fura-2-AM (Invitrogen). CMs were field stimulated at $1.0 \mathrm{~Hz}$ to determine transient amplitude, upstroke velocity, and rate of decay. All measurements were made following $>2$ min of conditioning of $1 \mathrm{~Hz}$-field stimuli to induce steady state. Transients were recorded at $1 \mathrm{~Hz}$. All $\mathrm{Ca}^{2+}$ transient/sarcomere dynamic data were analyzed using lonOptix lonWizard 6.3 (lonOptics Inc., Westwood, MA). Background fluorescence (Fbackground) for F380 and F340 were determined from cell-free regions. Data are expressed as F340/380 and were corrected for Fbackground.

\section{T-tubule quantification}

For T-tubule quantification we used dispersed CMs that were not reexposed to physiological calcium to preserve resting $\mathrm{CM}$ length. $\mathrm{CMs}$ were incubated in Di-8-ANEPPS ( $5 \mathrm{mM}$; ThermoFisher, cat\#D3167) for $5 \mathrm{~min}$, cells were rinsed in low-calcium relaxation buffer, and photographed on a Zeiss5Live laser scanning confocal microscope with a $\times 100$ oil immersion objective. Di-8-ANEPPS was excited at $488 \mathrm{~nm}$ and emission wavelengths $>505 \mathrm{~nm}$ were collected for an optical slice at a z-plane level without visible nuclei. Photomicrographs were analyzed for T-tubule organization using the AutoTT program designed by Drs. Guo and Song ${ }^{52}$. AutoTT allows simultaneous measurement of the longitudinally oriented and transversely oriented T-tubules in each of the ANEPPS-labeled CMs. To visualize $\mathrm{z}$-discs, isolated ventricular CMs were fixed in 4\% PFA for $10 \mathrm{~min}$ at $37^{\circ} \mathrm{C}$, and washed with PBS, permeabilized, and centrifuged at 300 RPM for $3 \mathrm{~min}$. Collected cells were stained with mouse anti a-actinin (Sigma A7811, lot 111M4845, 1:500 dilution), and visualized with Alexa Flour 594 donkey anti-mouse IgG (ThermoFisher, cat. A21203, lot 1722995, 1:500 dilution). Confocal Z-series stacks (step size $210 \mathrm{~nm}$ ) were acquired on a Zeiss confocal laser scanning microscope (LSM) 880 using the Airyscan detector, a Plan-Apochromat $\times 63 / 1.4$ DIC oil immersion objective. The laser wavelength and strength was $594 \mathrm{~nm}$ at $0.7 \%$ with a pinhole size of 2.59 AiryUnits (AU). The Zeiss Zen (black edition) software was used to detect Alexa Flour 594 in the Airyscan super resolution mode using main dichroic beam splitters $488 / 549$ and 405 . Raw.czi files were processed into deconvolved images using the Zen software. 3D reconstruction images were visualized and captured as.tif files in Zen blue version 3.4.89. Movies are a 50-frame series of images, 360-degree panorama.

\section{Cardiomyocyte surface area and nucleation measurement}

Isolated ventricular CMs were fixed in $4 \%$ PFA for $10 \mathrm{~min}$ at $37^{\circ} \mathrm{C}$ and washed once with PBS by centrifugation at 300 RPM for $3 \mathrm{~min}$. Collected cells were stained with DAPI at 1:10,000 dilution for $5 \mathrm{~min}$ at $37^{\circ} \mathrm{C}(\mathrm{H} 3570$, Thermo Fisher Scientific, Waltham, MA) following by another PBS wash prior to mounting on glass slides with ProLong ${ }^{\mathrm{Tm}}$ Gold Antifade mounting solution (Thermo Fisher, P36934). For surface area quantification, isolated CMs were examined using an Olympus IX-71 with DP72 color camera (12.8 megapixel cooled digital color camera). Measurement of CM size was performed using Olympus cellSens software area measuring function to trace the surface of CMs. For nucleation analysis, the number of DAPI stained nuclei/CM from different fields were quantified.

\section{Ploidy and nuclear volume measurement}

CM ploidy analyses were done as previously described with modifications $^{30}$. PFA fixed ventricular CMs from each group were used in ploidy and nuclear volume measurement. Confocal z-stacks of randomly selected CMs were captured using Nikon $\mathrm{Ti}, \mathrm{A} 1$ confocal microscope (Nikon, Japan) with a step size of $3 \mu \mathrm{m} . \times 40$ oil immersion objective was utilized for all acquisitions. Ploidy and nuclear volume were measured in z-stacks using Imaris Version 9 software (Bitplane AG, Switzerland). 3D Nuclei were identified and outlined with a standard threshold requirement for all groups using the Imaris cell analysis function to determine nuclear volume and mean DAPI intensity for ploidy analysis. Mean DAPI intensity of spleen cell nuclei within species were used as reference for diploid ( $2 \mathrm{~N}$ ) nuclei. CM nuclei were determined as diploid if their normalized intensity values were within 1-1.5 times range of reference cells. Nuclei were determined as tetraploid if their intensity values were within 1.5-2.5 times range of the reference cells. Polyploidy was assigned if DAPI intensity was greater than 2.5 times range of reference cells, as previously described ${ }^{53}$.

\section{Murine model of myocardial infarction}

MI surgery was performed as previously described ${ }^{50}$. In brief, we anesthetized mice with $1-3 \%$ isoflurane using a small animal vaporizer system. The pain reflex was examined to make sure that the mice were adequately anaesthetized before surgery. A thoracotomy was performed between the 4 th and 5 th ribs and the pericardial sac was removed. The heart was exposed and pushed out of the chest. The LAD coronary artery was identified under direct vision and was permanently ligated $3 \mathrm{~mm}$ below its origin using $6-0$ silk suture. After LAD ligation, the heart was returned to the intrathoracic space, the muscles were closed, and the skin was sutured using 4-0 prolene running sutures.

\section{Echocardiography}

A heating pad and rectal temperature probe were utilized to keep body temperature at $37{ }^{\circ} \mathrm{C}$ during the experiment. Modified parasternal long-axis and short-axis was utilized to determine left ventricular function and volume in M-mode, two-dimensional and Doppler echocardiography modes. We also used M-mode tracings at the mid-papillary level to estimate the systolic and diastolic parameters, and Teichholz formula at end-systole and end-diastole to measure the left ventricle (LV) volumes. All animals were anaesthetized using 1-3\% isoflurane during Echocardiography to maintain a heart rate of 450-500 BPM for all echocardiographic acquisitions. Echo was performed at baseline and at D2, D7, D14, D30, and D50 post-MI.

\section{Cardiac magnetic resonance imaging}

Cardiac magnetic resonance imaging (CMR) was performed on a 7-Tesla ClinScan system (Bruker, Ettlingen, Germany, http://www.bruker.com) equipped with a 4-element phased-array cardiac coil and a gradient system with a maximum strength of $450 \mathrm{mT} / \mathrm{m}$ and a maximum slew rate of $4500 \mathrm{mT} / \mathrm{m} / \mathrm{s}$. We acquired whole short-axis stack images from base to apex for comparison with late enhancement images. The short-axis images were planned perpendicular to the four-chamber long-axis image. For late gadolinium-enhanced magnetic resonance imaging, a $0.6 \mathrm{mmol} / \mathrm{kg}$ bolus of gadolinium-diethylenetriamine pentaacetic acid (Gd-DTPA; Gadavist, Bayer Health Care, Whippany, NJ) was injected using the intraperitoneal route. Imaging was initiated 10 min after the injection of Gd-DTPA using an electrocardiographically gated segmented magnetization-prepared fast low-angle shot sequence with a fixed inversion time at $500 \mathrm{~ms}$. CMR was performed at D3 and D14 post-MI. The CMR data were analyzed using commercially available post-processing software: CMR42 (Circle Cardiovascular Imaging, Calgary, Alberta, Canada, http://www.circlecvi.com) and ImageJ (NIH). Infarct length was calculated as a percentage of left ventricular length. All analyses were performed by blinded investigators.

\section{Statistics}

Values are expressed as mean \pm standard error of mean (s.e.m). We used student $T$-test, one-way-ANOVA, repeated-measures ANOVA and mixedeffects ANOVA with Tukey's corrections to compare data across species as appropriate. The mixed-effects model integrates analysis that uses the available data to estimate model parameters. Animals with partial data contribute to the estimation of some model parameters but not others. It is important to note that relying solely on repeated-measures ANOVA will limit the sample size to only animals that survived for the entire duration of the study and could lead to selection bias as we are only selecting a subgroup of animals. Throughout the manuscript, analyses that compared species and time but did not include paired samples such as data in Fig. $4 \mathrm{~g}$, $\mathrm{h}$ were analyzed using 2-way independent ANOVA. Sample sizes, statistical tests and $P$ values are indicated in the figures or figure legends. Animal numbers are presented as a range based on the number of animals included in each analysis. These numbers are included in the figure legends. Throughout the analyses, a $P$ value $<0.05$ was considered statistically significant.

\section{Reporting summary}

Further information on research design is available in the Nature Research Reporting Summary linked to this article. 


\section{DATA AVAILABILITY}

The authors declare that all the data supporting the findings of this study are available within the paper and are contained within Supplementary Information.

Received: 12 January 2021; Accepted: 21 October 2021; Published online: 17 November 2021

\section{REFERENCES}

1. Benjamin, E. J. et al. Heart Disease and Stroke Statistics-2017 Update: A Report From the American Heart Association. Circulation 135, e146-e603 (2017).

2. Iwakura, K. Modulation of individual susceptibility to the no-reflow phenomenon after acute myocardial infarction. Curr. Pharm. Des. 19, 4519-4528 (2013).

3. McAloon, C. J. et al. The changing face of cardiovascular disease 2000-2012: An analysis of the world health organisation global health estimates data. Int J. Cardiol. 224, 256-264 (2016)

4. Pfeffer, M. A. \& Braunwald, E. Ventricular remodeling after myocardial infarction. Experimental observations and clinical implications. Circulation 81, 1161-1172 (1990).

5. Jugdutt, B. I. Ventricular remodeling after infarction and the extracellular collagen matrix: when is enough enough? Circulation 108, 1395-1403 (2003).

6. Struthers, A. D. Pathophysiology of heart failure following myocardial infarction. Heart 91, ii14-ii16 (2005).

7. Marin-Juez, R. et al. Coronary revascularization during heart regeneration is regulated by epicardial and endocardial cues and forms a scaffold for cardiomyocyte repopulation. Dev. Cell 51, 503-515.e504 (2019).

8. Bader, D. \& Oberpriller, J. O. Repair and reorganization of minced cardiac Muscle in the adult newt (NotophthalMus viridescens). J. Morphol. 155, 349-357 (1978).

9. Flink, I. L. Cell cycle reentry of ventricular and atrial cardiomyocytes and cells within the epicardium following amputation of the ventricular apex in the axolotl, Amblystoma mexicanum: confocal microscopic immunofluorescent image analysis of bromodeoxyuridine-labeled nuclei. Anat. Embryol. 205, 235-244 (2002).

10. Poss, K. D., Wilson, L. G. \& Keating, M. T. Heart regeneration in zebrafish. Science 298, 2188-2190 (2002).

11. Chablais, F. \& Jazwinska, A. The regenerative capacity of the zebrafish heart is dependent on TGFbeta signaling. Development 139, 1921-1930 (2012).

12. Porrello, E. R. et al. Transient regenerative potential of the neonatal mouse heart. Science 331, 1078-1080 (2011).

13. Haughton, C. L., Gawriluk, T. R. \& Seifert, A. W. The Biology and Husbandry of the African Spiny Mouse (Acomys cahirinus) and the Research Uses of a Laboratory Colony. J. Am. Assoc. Lab Anim. Sci. 55, 9-17 (2016).

14. Harn, H. I. et al. Symmetry breaking of tissue mechanics in wound induced hair follicle regeneration of laboratory and spiny mice. Nat. Commun. 12, 2595 (2021).

15. Brant, J. O., Yoon, J. H., Polvadore, T., Barbazuk, W. B. \& Maden, M. Cellular events during scar-free skin regeneration in the spiny mouse, Acomys. Wound Repair Regen. 24, 75-88 (2016).

16. Gawriluk, T. R. et al. Comparative analysis of ear-hole closure identifies epimorphic regeneration as a discrete trait in mammals. Nat. Commun. 7, 11164 (2016).

17. Jiang, T. X., Harn, H. I., Ou, K. L., Lei, M. \& Chuong, C. M. Comparative regenerative biology of spiny (Acomys cahirinus) and laboratory (Mus Musculus) mouse skin. Exp. Dermatol 28, 442-449 (2019).

18. Matias Santos, D. et al. Ear wound regeneration in the African spiny mouse Acomys cahirinus. Regeneration 3, 52-61 (2016).

19. Seifert, A. W. et al. Skin shedding and tissue regeneration in African spiny mice (Acomys). Nature 489, 561-565 (2012).

20. Okamura, D. M. et al. Scarless repair of acute and chronic kidney injury in African Spiny mice (Acomys cahirinus). bioRxiv 315069 (2018).

21. Hirose, K. et al. Evidence for hormonal control of heart regenerative capacity during endothermy acquisition. Science 364, 184-188 (2019).

22. Qi, Y. et al. Functional heart recovery in an adult mammal, the spiny mouse. Int J. Cardiol. 338, 196-203 (2021).

23. Carmeliet, P. \& Jain, R. K. Molecular mechanisms and clinical applications of angiogenesis. Nature 473, 298-307 (2011).

24. Potente, M., Gerhardt, H. \& Carmeliet, P. Basic and therapeutic aspects of angiogenesis. Cell 146, 873-887 (2011).

25. Patterson, M. et al. Frequency of mononuclear diploid cardiomyocytes underlies natural variation in heart regeneration. Nat. Genet 49, 1346-1353 (2017).

26. Salimova, E. et al. Variable outcomes of human heart attack recapitulated in genetically diverse mice. NPJ Regen. Med 4, 5 (2019).

27. Alkass, K. et al. No evidence for cardiomyocyte number expansion in preadolescent mice. Cell 163, 1026-1036 (2015).
28. Soonpaa, M. H., Kim, K. K., Pajak, L., Franklin, M. \& Field, L. J. Cardiomyocyte DNA synthesis and binucleation during murine development. Am. J. Physiol. 271, H2183-H2189 (1996).

29. Lozoya, O. A., Gilchrist, C. L. \& Guilak, F. Universally conserved relationships between nuclear shape and cytoplasmic mechanical properties in human stem cells. Sci. Rep. 6, 23047 (2016).

30. Broughton, K. M. et al. Cardiac interstitial tetraploid cells can escape replicative senescence in rodents but not large mammals. Commun. Biol. 2, 205 (2019).

31. Sipido, K. R., Carmeliet, E. \& Van de Werf, F. T-type Ca2+ current as a trigger for $\mathrm{Ca} 2+$ release from the sarcoplasmic reticulum in guinea-pig ventricular myocytes. J. Physiol. 508, 439-451 (1998).

32. Nuss, H. B. \& Houser, S. R. T-type Ca2+ current is expressed in hypertrophied adult feline left ventricular myocytes. Circ. Res. 73, 777-782 (1993).

33. Schroder, E., Byse, M. \& Satin, J. L-type calcium channel $C$ terminus autoregulates transcription. Circ. Res 104, 1373-1381 (2009).

34. Dick, S. A. et al. Self-renewing resident cardiac macrophages limit adverse remodeling following myocardial infarction. Nat. Immunol. 20, 29-39 (2019).

35. Epelman, S., Liu, P. P. \& Mann, D. L. Role of innate and adaptive immune mechanisms in cardiac injury and repair. Nat. Rev. Immunol. 15, 117-129 (2015).

36. Lindsey, M. L. et al. Guidelines for experimental models of myocardial ischemia and infarction. Am. J. Physiol. Heart Circ. Physiol. 314, H812-H838 (2018).

37. Heusch, G. The coronary circulation as a target of cardioprotection. Circ. Res 118, 1643-1658 (2016).

38. Wang, J., Toan, S. \& Zhou, H. Mitochondrial quality control in cardiac microvascular ischemia-reperfusion injury: new insights into the mechanisms and therapeutic potentials. Pharm. Res. 156, 104771 (2020).

39. Kloner, R. A. No-reflow phenomenon: maintaining vascular integrity. J. Cardiovasc Pharm. Ther. 16, 244-250 (2011).

40. Nazir, S. A. et al. Strategies to attenuate micro-vascular obstruction during P-PCl: the randomized reperfusion facilitated by local adjunctive therapy in ST-elevation myocardial infarction trial. Eur. Heart J. 37, 1910-1919 (2016).

41. Zhang, S. et al. Comparison of various niches for endothelial progenitor cell therapy on ischemic myocardial repair: coexistence of host collateralization and Akt-mediated angiogenesis produces a superior microenvironment. Arterioscler Thromb. Vasc. Biol. 32, 910-923 (2012).

42. Simkin, J., Gawriluk, T. R., Gensel, J. C. \& Seifert, A. W. Macrophages are necessary for epimorphic regeneration in African spiny mice. Elife 6, e24623 (2017).

43. Ostadal, B., Schiebler, T. H. \& Rychter, Z. Relations between development of the capillary wall and myoarchitecture of the rat heart. Adv. Exp. Med. Biol. 53, 375-388 (1975).

44. Fitzpatrick, J. R. 3rd et al. Tissue-engineered pro-angiogenic fibroblast scaffold improves myocardial perfusion and function and limits ventricular remodeling after infarction. J. Thorac. Cardiovasc. Surg. 140, 667-676 (2010).

45. Kikuchi, K. et al. Primary contribution to zebrafish heart regeneration by gata4(+) cardiomyocytes. Nature 464, 601-605 (2010).

46. Oberpriller, J. O. \& Oberpriller, J. C. Response of the adult newt ventricle to injury. J. Exp. Zool. 187, 249-253 (1974).

47. Jopling, $C$. et al. Zebrafish heart regeneration occurs by cardiomyocyte dedifferentiation and proliferation. Nature 464, 606-609 (2010).

48. Windmueller, R. et al. Direct comparison of mononucleated and binucleated cardiomyocytes reveals molecular mechanisms underlying distinct proliferative competencies. Cell Rep 30, 3105-3116.e3104 (2020).

49. Cribbs, L. L. et al. Identification of the t-type calcium channel $(\mathrm{Ca}(\mathrm{v}) 3.1 \mathrm{~d})$ in developing mouse heart. Circ. Res 88, 403-407 (2001).

50. Al-Darraji, A. et al. Azithromycin therapy reduces cardiac inflammation and mitigates adverse cardiac remodeling after myocardial infarction: potential therapeutic targets in ischemic heart disease. PLoS One 13, e0200474 (2018).

51. Ahern, B. M. et al. Myocardial-restricted ablation of the GTPase RAD results in a pro-adaptive heart response in mice. J. Biol. Chem. 294, 10913-10927 (2019).

52. Guo, A. \& Song, L.S. AutoTT: automated detection and analysis of T-tubule architecture in cardiomyocytes. Biophys. J. 106, 2729-2736 (2014).

53. Wu, Y. et al. LRP6 downregulation promotes cardiomyocyte proliferation and heart regeneration. Cell Res. 31, 450-462 (2021).

\section{ACKNOWLEDGEMENTS}

The authors would like to thank Josh Sarli for his help with Acomys husbandry and Thomas Wilkop Ph.D at the microscopy core at the University of Kentucky for his assistance with imaging. We thank the Markey Cancer Center and the COBRE histology core for their assistance with the tissue specimen preparation. We thank Binoy Joseph for his assistance in imaging and the Spinal Cord and Brain Injury Research Center for use of their imaging equipment. We thank Beverly Meacham and the University of Kentucky MRISC center for the assistance with cardiac MRI studies. A.A.L. is supported by NIH Grant R01 HL124266. Work in A.W.S.'s lab is supported by $\mathrm{NIH}$ R01 AR070313. The content in this article is solely the responsibility of the 
authors and does not necessarily represent the official views of the National Institutes of Health. Markey Cancer Center Core: This research was supported by the Biospecimen Procurement and Translational Pathology Shared Resource Facility of the University of Kentucky Markey Cancer Center (P30CA177558). COBRE Core: Research reported in this publication was supported by an Institutional Development Award (IDeA) from the National Institute of General Medical Sciences of the National Institutes of Health under grant number P30 GM127211.

\section{AUTHOR CONTRIBUTIONS}

J.S., A.W.S., A.A.L., K.S., H.P. and R.R.D. designed and had oversight of the project and experiments. K.S., H.P., R.R.D., E.G., B.M.A., B.M.L., D.P., H.T., G.A.E. and A.N. performed all the experiments. K.S., H.P., R.R.D., B.M.A., B.M.L., J.S., A.W.S., A.A.L. analyzed the data. K.S., H.P., J.S., A.W.S., A.A.L. wrote and edited the manuscript. H.P. and K.S. contributed equally to the study. J.S., A.W.S. and A.A.L. equally supervised the study. All authors commented on and edited the final version.

\section{COMPETING INTERESTS}

The authors declare no competing interests.

\section{ADDITIONAL INFORMATION}

Supplementary information The online version contains supplementary material available at https://doi.org/10.1038/s41536-021-00186-4.
Correspondence and requests for materials should be addressed to Jonathan Satin, Ashley W. Seifert or Ahmed Abdel-Latif.

Reprints and permission information is available at http://www.nature.com/ reprints

Publisher's note Springer Nature remains neutral with regard to jurisdictional claims in published maps and institutional affiliations. Attribution 4.0 International License, which permits use, sharing, adaptation, distribution and reproduction in any medium or format, as long as you give appropriate credit to the original author(s) and the source, provide a link to the Creative Commons license, and indicate if changes were made. The images or other third party material in this article are included in the article's Creative Commons license, unless indicated otherwise in a credit line to the material. If material is not included in the article's Creative Commons license and your intended use is not permitted by statutory regulation or exceeds the permitted use, you will need to obtain permission directly from the copyright holder. To view a copy of this license, visit http://creativecommons. org/licenses/by/4.0/.

(c) The Author(s) 2021 